ARTICLE

\title{
The mTORC1/4E-BP1 axis represents a critical signaling node during fibrogenesis
}

Hannah V. Woodcock¹, Jessica D. Eley', Delphine Guillotin', Manuela Platé (1) ', Carmel B. Nanthakumar², Matteo Martufi ${ }^{3}$, Simon Peace ${ }^{2}$, Gerard Joberty ${ }^{4}$, Daniel Poeckel ${ }^{4}$, Robert B. Good², Adam R. Taylor ${ }^{2}$,

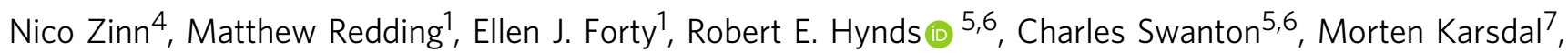
Toby M. Maher ${ }^{8}$, Andrew Fisher ${ }^{9}$, Giovanna Bergamini ${ }^{4}$, Richard P. Marshall ${ }^{2}$, Andy D. Blanchard ${ }^{2}$, Paul F. Mercer ${ }^{1} \&$ Rachel C. Chambers (i) ${ }^{1}$

Myofibroblasts are the key effector cells responsible for excessive extracellular matrix deposition in multiple fibrotic conditions, including idiopathic pulmonary fibrosis (IPF). The $\mathrm{PI3K} / \mathrm{Akt} / \mathrm{mTOR}$ axis has been implicated in fibrosis, with pan-PI3K/mTOR inhibition currently under clinical evaluation in IPF. Here we demonstrate that rapamycin-insensitive mTORC1 signaling via 4E-BP1 is a critical pathway for TGF- $\beta_{1}$ stimulated collagen synthesis in human lung fibroblasts, whereas canonical PI3K/Akt signaling is not required. The importance of mTORC1 signaling was confirmed by CRISPR-Cas9 gene editing in normal and IPF fibroblasts, as well as in lung cancer-associated fibroblasts, dermal fibroblasts and hepatic stellate cells. The inhibitory effect of ATP-competitive mTOR inhibition extended to other matrisome proteins implicated in the development of fibrosis and human disease relevance was demonstrated in live precision-cut IPF lung slices. Our data demonstrate that the mTORC1/4E-BP1 axis represents a critical signaling node during fibrogenesis with potential implications for the development of novel anti-fibrotic strategies.

\footnotetext{
${ }^{1}$ Centre for Inflammation and Tissue Repair, UCL Respiratory, Rayne Building, University College London, London WC1E 6JF, UK. ${ }^{2}$ Fibrosis Discovery Performance Unit, Respiratory Therapy Area, Medicines Research Centre, GlaxoSmithKline R\&D, Gunnels Wood Road, Stevenage SG1 2NY, UK. ${ }^{3}$ Target Sciences, Medicines Research Centre, GlaxoSmithKline R\&D, Gunnels Wood Road, Stevenage SG1 2NY, UK. ${ }^{4}$ Cellzome, a GSK Company, Meyershofstrasse 1, 69117 Heidelberg, Germany. ${ }^{5}$ CRUK Lung Cancer Centre of Excellence, UCL Cancer Institute, University College London, London WC1E 6DD, UK. ${ }^{6}$ Cancer Evolution and Genome Instability Laboratory, The Francis Crick Institute, London NW1 1AT, UK. ${ }^{7}$ Nordic Bioscience, Herlev 2730 , Denmark. ${ }^{8}$ Fibrosis Research Group, Inflammation, Repair \& Development Section, NHLI, Imperial College, London SW3 6LY, UK. ${ }^{9}$ Newcastle Fibrosis Research Group, Newcastle University Translational and Clinical Research Institute, Newcastle upon Tyne, UK. These authors contributed equally: Hannah V. Woodcock, Jessica D. Eley. Correspondence and requests for materials should be addressed to R.C.C. (email: r.chambers@ucl.ac.uk)
} 
$\mathrm{F}$ ibrosis, defined as the abnormal accumulation of extracellular matrix (ECM), is a pathological feature of many chronic inflammatory and metabolic diseases and is often closely linked with organ dysfunction and, ultimately, organ failure $^{1,2}$. The importance of the stroma in influencing cancer progression is also gaining increasing recognition ${ }^{1,3}$. Despite this high unmet clinical need, only two anti-fibrotic drugs, Pirfenidone/Esbriet ${ }^{\circledR}$ and Nintedanib/Ofev ${ }^{\circledR}$ have been approved to date. Moreover, these agents slow rather than halt disease progression in idiopathic pulmonary fibrosis (IPF) ${ }^{4,5}$, the most rapidly progressive and fatal of all fibrotic conditions. The underlying etiology of IPF remains poorly understood although current evidence suggests this condition likely arises as a result of a highly dysregulated wound healing response following chronic epithelial injury on the background of a combination of genetic predisposition and environmental factors (including cigarette smoking) and cellular senescence associated with ageing ${ }^{6-8}$. Highly synthetic and $a$-smooth muscle actin positive myofibroblasts are regarded the key effector cells of the fibrogenic response during both normal wound healing and in the context of pathological fibrosis ${ }^{9}$, including IPF ${ }^{10-13}$. The persistence of these cells, as a result of a failure in apoptosis, is felt to be a key event in the initiation and progression of fibrosis ${ }^{14}$. In terms of key mediators involved in promoting excessive myofibroblast differentiation and fibrogenesis, current evidence points to a key role for the pleiotropic cytokine, transforming growth factor- $\beta$ (in particular the TGF- $\beta_{1}$ isoform), in multiple fibrotic conditions ${ }^{15}$. TGF- $\beta_{1}$ signals through the canonical Smad pathway and several noncanonical pathways to influence cellular function in a cell-specific and cell-context dependent manner. Therapeutic strategies aimed at targeting the dysregulated TGF- $\beta_{1}$ axis in fibrosis, without compromising its critical roles in tissue and immune homeostasis, are being intensely pursued ${ }^{16}$.

The phosphoinositide-3-kinase (PI3K)/mechanistic target of rapamycin (mTOR) signaling pathway plays a central role in regulating a broad range of fundamental cellular processes, including metabolism, cell cycle progression, proliferation, growth, autophagy, and protein synthesis ${ }^{17}$. Activation of class 1 PI3K results in the production of membrane-localized phosphatidylinositol-3,4,5-trisphosphate $\left(\mathrm{PIP}_{3}\right)$ and recruitment of Akt via its pleckstrin homology domain. mTOR functions at two distinct nodes in this signaling axis. mTOR complex 2 (mTORC2) and 3-phosphoinositide-dependent protein kinase-1 (PDK1) phosphorylate Akt at the plasma membrane to stabilize the catalytic site of Akt for maximal activation ${ }^{18}$. Once activated, Akt phosphorylates the TSC2 subunit of the tuberous sclerosis complex (TSC), a key control switch for mTORC1. Phosphorylation and inhibition of TSC2 lead to the accumulation of GTPbound RAS homologue enriched in brain (Rheb) and activation of mTORC1 signaling via several downstream substrates, including p70S6K and eukaryotic translation initiation factor 4Ebinding protein $1(4 \mathrm{E}-\mathrm{BP} 1)^{19}$.

The PI3K/mTOR pathway has previously been implicated in influencing fibroblast proliferative responses and TGF- $\beta_{1}$-induced myofibroblast differentiation and collagen production ${ }^{20,21}$. More recently, we provided a strong scientific rationale for progressing the potent pan-PI3 kinase/mTOR inhibitor Omipalisib (GSK2126458) as a novel anti-fibrotic agent in a proof-ofmechanism trial in IPF (https://clinicaltrials.gov/ct2/show/ NCT01725139) ${ }^{22}$. Omipalisib displays broad target specificity and may overcome functional redundancy between PI3K isoforms and compensatory feedback loops in this pathway but ontarget-toxicities associated with this class of inhibitors could be limiting $23-25$.

The mechanism by which the $\mathrm{PI} 3 \mathrm{~K} / \mathrm{mTOR}$ pathway regulates TGF- $\beta_{1}$-induced collagen synthesis is poorly understood. In this study, we characterize a unique toolbox of commercially available and proprietary pharmacological inhibitors and use these in combination with CRISPR-Cas9 gene editing and siRNA approaches to deconvolute the mechanism by which pan-PI3 kinase/mTOR inhibition blocks TGF- $\beta_{1}$-induced collagen synthesis in primary human lung fibroblasts (pHLFs). We show that the potent fibrogenic effects of TGF- $\beta_{1}$ are mediated via the cooperation between canonical Smad3 and rapamycin-insensitive mTORC1/4E-BP1 signaling and that the canonical PI3K-Akt axis is dispensable for this response. Furthermore, ATP-competitive mTOR inhibition halts collagen synthesis in live unmanipulated precision-cut IPF lung slices. The critical role of the mTORC1/ $4 \mathrm{E}-\mathrm{BP} 1$ axis in influencing collagen synthesis is further generalizable to fibrogenic cells implicated in the development of fibrosis in the liver and skin, as well as the stromal reaction in lung cancer. Taken together, these experiments shed light on the signaling pathways by which TGF- $\beta_{1}$ exerts its potent fibrogenic effects and provide support for selectively targeting mTORC1 signaling in IPF and potentially other fibrotic conditions.

\section{Results}

TGF- $\beta_{1}$-induced PI3K and mTOR signaling in lung fibroblasts. Initial studies, aimed at defining the kinetics of PI3K/mTOR and canonical Smad signaling in pHLFs during TGF- $\beta_{1}$-induced collagen deposition, revealed that Smad signaling was rapid and relatively short-lived with $\mathrm{Smad} 2$ phosphorylation peaking within the first hour and declining after $2 \mathrm{~h}$ (Fig. 1a). This preceded mTORC1 signaling, as evidenced by $\mathrm{p} 70 \mathrm{~S} 6 \mathrm{~K}$ and $4 \mathrm{E}-\mathrm{BP} 1$ phosphorylation (Ser65), which was observed from $2 \mathrm{~h}$ onwards and was sustained for at least $12 \mathrm{~h}$. Maximal PDK1-dependent phosphorylation of Akt (Thr308) and mTORC2-dependent phosphorylation of its substrates Akt (Ser473) and SGK1 (inferred by phosphorylation of the SGK1 substrate $\mathrm{NDRG1}^{26}$ ) peaked $12 \mathrm{~h}$ post-stimulation.

PI3K pathway inhibition and the TGF- $\beta_{1}$ collagen response. We next investigated the contribution of the $\mathrm{PI} 3 \mathrm{~K} / \mathrm{mTOR}$ signaling axis to TGF- $\beta_{1}$-induced collagen synthesis by quantitative high-content imaging of collagen I deposition under macromolecular crowding conditions ${ }^{22,27}$. TGF- $\beta_{1}(1 \mathrm{ng} / \mathrm{ml})$ increased pHLF collagen deposition by 2.1 -fold $( \pm 0.1)$ after $72 \mathrm{~h}$ (Fig. 1b, c). To determine whether canonical PI3K signaling was necessary for this response, we investigated the effect of highly selective inhibitors of critical components of the PI3K/Akt axis: PI3K (Compound 2), Akt (MK2206), and PDK1 (GSK23344770) (Supplementary Fig. 1). A schematic of inhibitors used with their corresponding targets is shown in Fig. 2a. Compound 2, an ATPcompetitive inhibitor selective for class 1 PI3K isoforms that exhibits low affinity for mTOR (Supplementary Table 2), attenuated both Akt Thr308 and Ser473 phosphorylation in addition to inhibiting phosphorylation of downstream substrates of Akt (PRAS40 and GSK3 $\beta$; Fig. 2b). In contrast, this inhibitor had no effect on TGF- $\beta_{1}$-induced collagen deposition (Fig. $2 \mathrm{c}$ ). Similarly, treatment with either the allosteric inhibitor of Akt, MK2206, or the PDK1 inhibitor, GSK2334479, attenuated Akt phosphorylation at both Thr308 and Ser473 sites in addition to downstream Akt signaling (Fig. $2 \mathrm{~d}, \mathrm{f}$ ) but had no effect on the TGF- $\beta_{1}$-induced collagen response (Fig. 2e, g). The mTORC2 substrates, Akt and SGK1, require sequential phosphorylation by PDK1 and mTORC2 to become fully activated. TGF- $\beta_{1}$-induced mTORC2mediated downstream signaling (Akt and NDRG) was attenuated in the presence of a PDK1 inhibitor, but collagen synthesis was unaffected (Fig. 2f, g). Taken together, these data led us to 

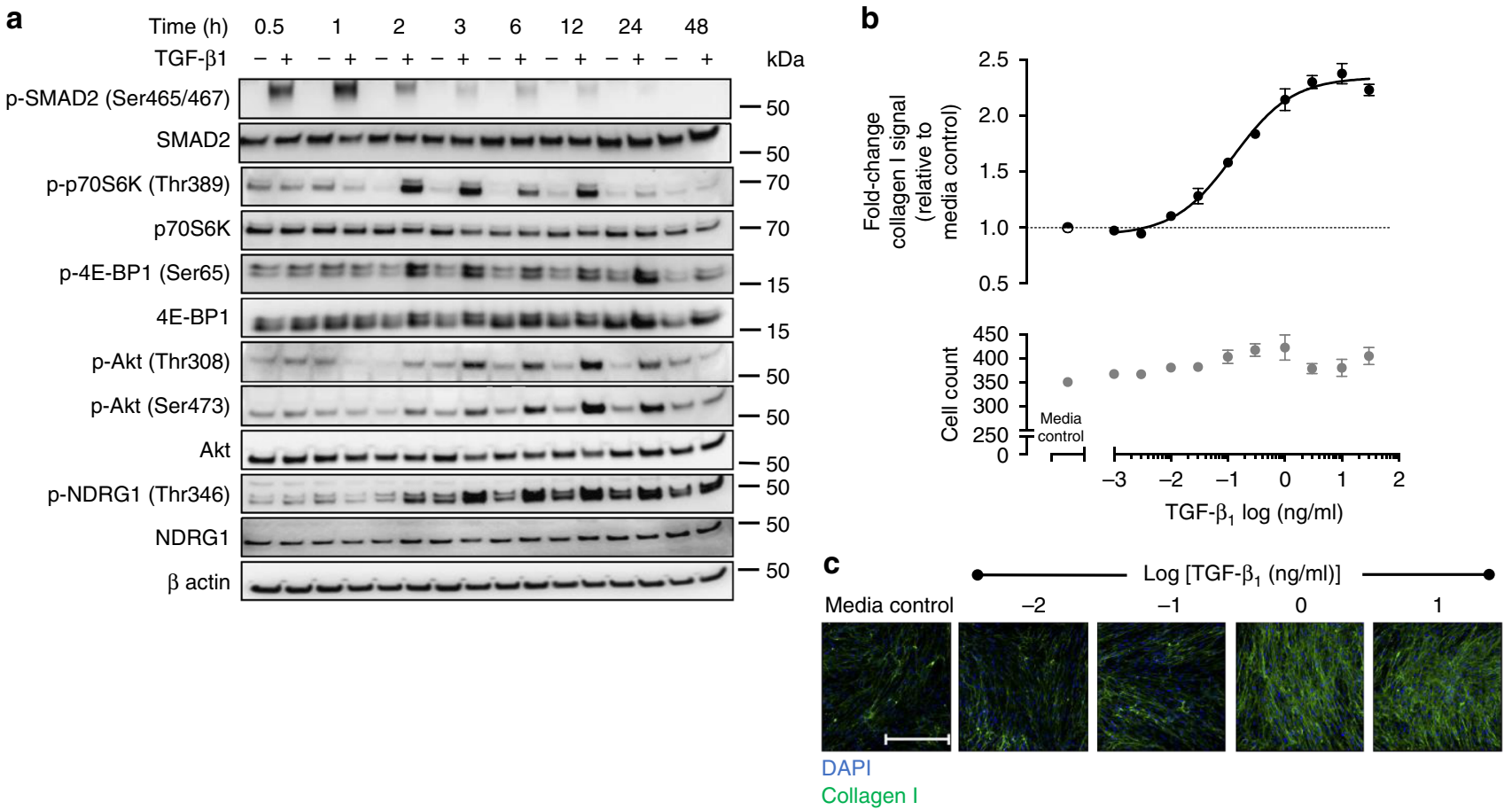

Fig. 1 TGF- $\beta_{1}$ induces Smad phosphorylation and mTOR signaling and upregulates collagen I deposition in control primary human lung fibroblasts. Control primary human lung fibroblasts ( $\mathrm{pHLFs}$ ) were stimulated with TGF- $\beta_{1}(1 \mathrm{ng} / \mathrm{ml})$ for $30 \mathrm{~min}$ to $48 \mathrm{~h}$ prior to cell lysis. Phosphorylation of specified proteins is shown by Western blot (a). Additionally, pHLFs were stimulated with increasing concentrations of TGF- $\beta_{1}(1 \mathrm{pg} / \mathrm{ml}$ to $30 \mathrm{ng} / \mathrm{ml})$ for $72 \mathrm{~h}$ with collagen I deposition assessed by macromolecular crowding assay (b). Data are expressed as the fold change in collagen I signal relative to the media control (4 field of view imaged per well) and cell counts obtained by staining nuclei with DAPI. Representative images are shown in c. Scale bars $=360 \mu$ m. Each data point shown is mean \pm SEM $(n=4)$ and is representative of 3 independent experiments

conclude that canonical PI3K/Akt signaling is redundant for TGF- $\beta_{1}$-induced collagen synthesis.

The role of mTOR signaling in the TGF- $\beta_{1}$ collagen response. Having ruled out a role for the linear PI3K/Akt pathway, we next investigated the contribution of mTOR signaling to TGF- $\beta_{1}$ induced collagen synthesis using four structurally distinct, potent and highly selective ATP-competitive mTOR kinase inhibitors that target both mTORC1 and mTORC2 (Supplementary Table 2, Supplementary Fig. 1). The first compound, AZD8055, attenuated TGF- $\beta_{1}$-induced phosphorylation of the mTORC1 substrates, p70S6K (Thr389) and 4E-BP1 (Thr37/46, Ser65), as well as the mTORC2 substrates, Akt (Ser473) and SGK1 (phosphorylated NDRG1 Thr346) in a concentration-dependent manner (Fig. 3a). This compound was also highly effective at inhibiting TGF- $\beta_{1}$ induced collagen synthesis (Fig. $3 \mathrm{~b}, \mathrm{c}$ ). This observation was reproducible in fibroblasts derived from 4 additional donors (Supplementary Table 3). Similar inhibitory profiles were observed with the ATP-competitive mTOR inhibitors; Torin-1 (Fig. 3d and Supplementary Fig. 3a), Compound 1 (Fig. 3e and Supplementary Fig. 3b), and CZ415 (Fig. 3f and Supplementary Fig. 2). Supplementary Table 4 illustrates the comparative selectivity profiles of CZ415 and AZD8055 derived from the MS-based binding analysis. The complete selectivity profiles are available in Supplementary Data 1. AZD8055 also significantly attenuated the peak increase in COL1A1 mRNA levels following TGF- $\beta_{1}$ stimulation suggesting that mTOR signaling acts, at least in part, by influencing COL1A1 mRNA levels (Fig. 3g).

Having established a key role for mTOR in mediating the fibrogenic effects of TGF- $\beta_{1}$, we next investigated the relative contributions of mTORC1 and mTORC2 by CRISPR-Cas9 gene editing of either RPTOR (mTORC1) or RICTOR (mTORC2).
Successful gene editing was confirmed by Western blot (Fig. 3h). TGF- $\beta_{1}$-induced collagen synthesis was maintained in RICTORedited cells (Fig. $3 \mathrm{i}, \mathrm{j}$ ). In contrast, RPTOR gene editing resulted in near complete inhibition of TGF- $\beta_{1}$-induced collagen synthesis. These observations were confirmed in a second assay based on the quantitation of hydroxyproline in procollagen by reverse phase HPLC (Fig. 3k) and provided strong evidence that mTORC1 is a key signaling node involved in mediating the potent fibrogenic effects of TGF- $\beta_{1}$.

The PI3K pathway is considered to be the primary pathway for the activation of mTORC1 (Fig. 2a). Akt phosphorylates TSC2 at multiple sites to relieve the inhibition on Rheb and stimulate mTORC1 activity. However, neither PI3K nor Akt inhibition influenced mTORC1 downstream signaling despite attenuating TSC2 phosphorylation at the Akt-dependent Ser939 residue (Supplementary Fig. 4a, b). MAPK signaling via ERK represents a parallel pathway which also leads to the phosphorylation of TSC2 ${ }^{19}$. Phosphorylation of TSC2 at the ERK-dependent Ser664 residue was retained when fibroblasts were stimulated with TGF- $\beta_{1}$ in the presence of inhibitors targeting PI3K, Akt, or PDK1 (Supplementary Fig. 4b). In order to rule out the possibility of ERK-mediated compensation when the Akt pathway is fully inhibited, we show that the ERK inhibitor AS-703026, used either on its own or in combination with the Akt inhibitor (MK2206), did not attenuate TGF- $\beta_{1}$-induced collagen deposition (Supplementary Fig. 4c, d). Inhibition of RSK1 (SL0101), which lies downstream of ERK and also phosphorylates TSC2, similarly did not inhibit the TGF- $\beta_{1}$ collagen response (Supplementary Fig. 4e). This led us to conclude that TGF- $\beta_{1}$-induced collagen synthesis is not dependent on Aktor MAPK-mediated phosphorylation of TSC2.

Canonical Smad signaling was found to precede the activation of mTORC1 signaling in response to TGF- $\beta_{1}$ stimulation (Fig. 1a), 
a

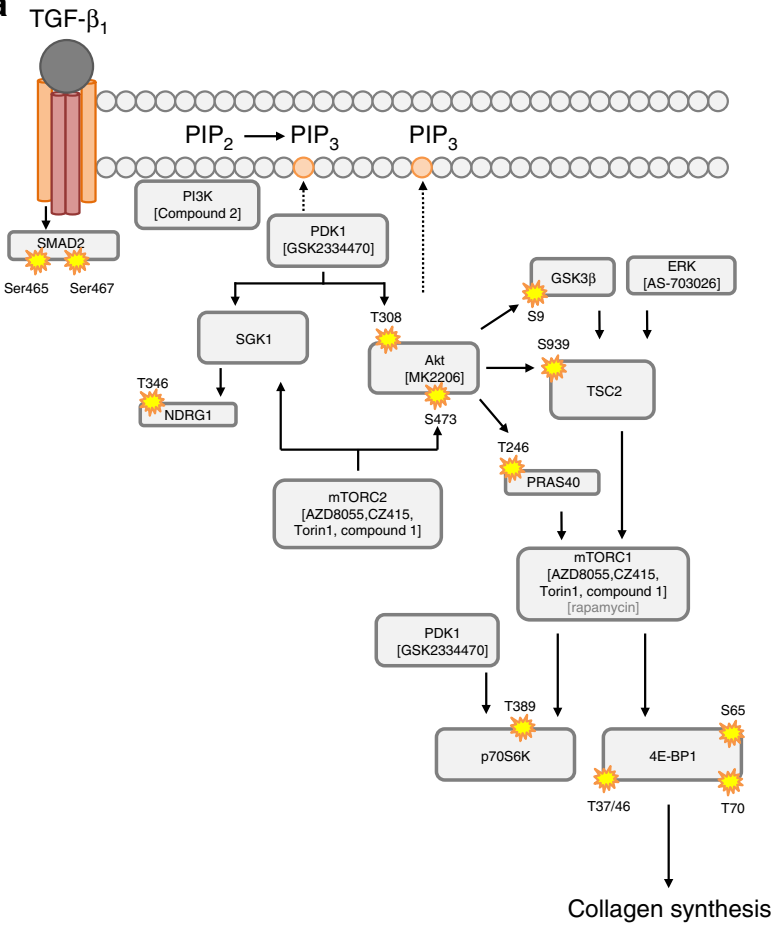

d

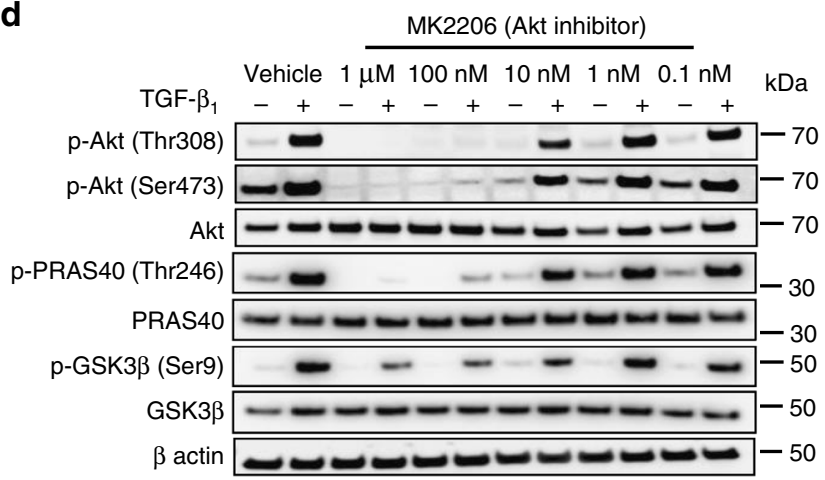

$\mathbf{f}$ b

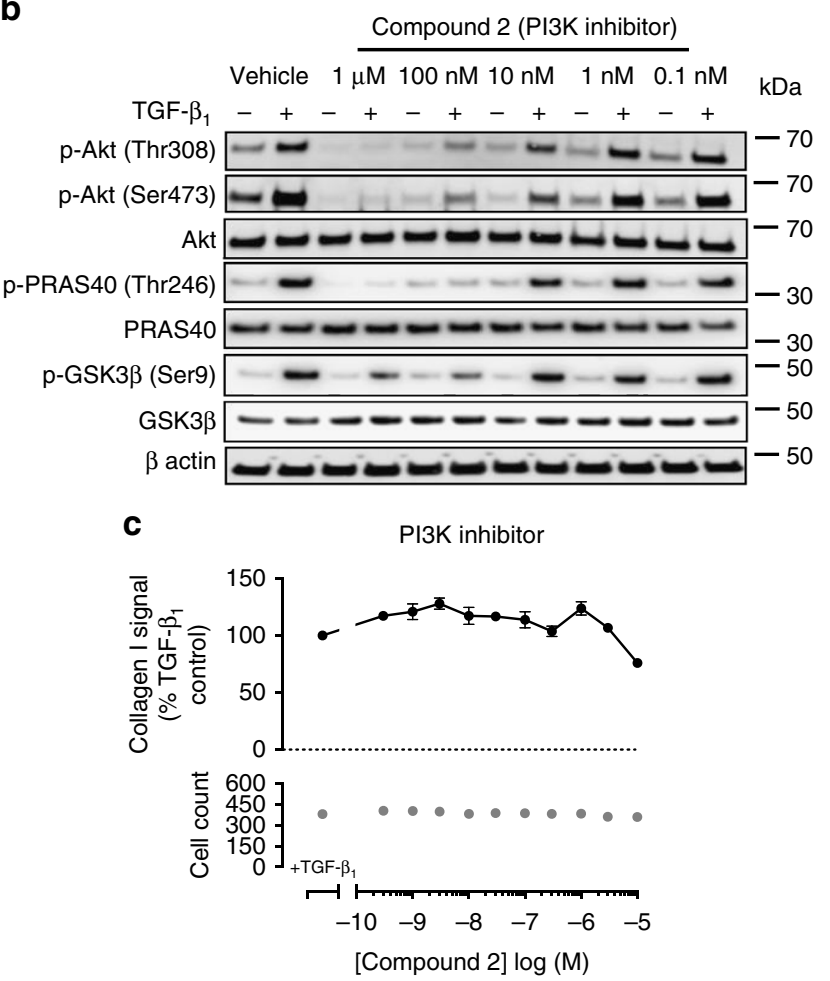

e

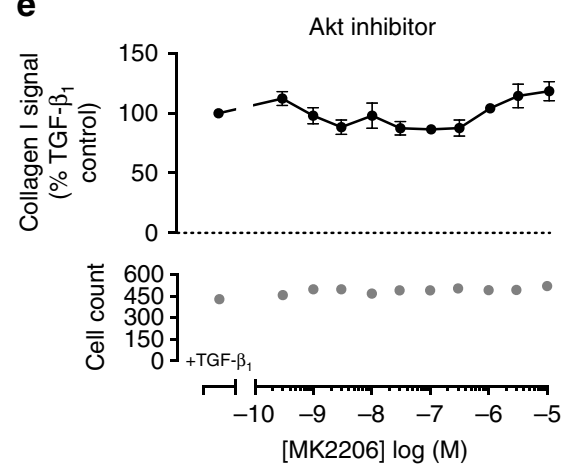

g

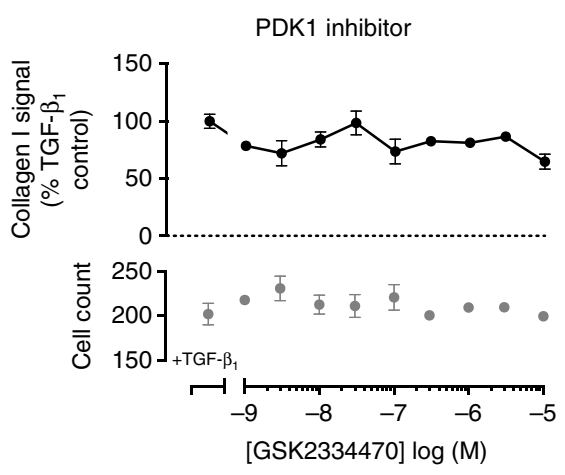

Fig. 2 Inhibition of TGF- $\beta_{1}$-induced PI3K, Akt, and PDK-1 signaling has no effect on collagen I deposition in pHLFs. A schematic, generated by author J.D.E., of inhibitors and their corresponding target is shown in a. pHLFs were pre-incubated with vehicle ( $0.1 \%$ DMSO) or increasing concentrations of Compound 2, MK2206 or $1 \mu$ M GSK2334470 prior to stimulation with TGF- $\beta_{1}(1 \mathrm{ng} / \mathrm{ml})$. Cells were lysed $12 \mathrm{~h}$ later and the phosphorylation of specified proteins was assessed by Western blot $(\mathbf{b}, \mathbf{d}, \mathbf{f})$. Data are representative of 3 independent experiments. Additionally, pHLFs were pre-incubated with vehicle ( $0.1 \%$ DMSO) or increasing concentrations of Compound 2 (c), MK2206 (e), or GSK2334470 (g) and stimulated with TGF- $\beta_{1}(1 \mathrm{ng} / \mathrm{ml})$ for $72 \mathrm{~h}$ with collagen deposition assessed by macromolecular crowding assay. Data are expressed as collagen I signal calculated as a percentage of the TGF- $\beta_{1}$-treated control ( $n=4$ fields of view imaged per well) and cell counts obtained by staining nuclei with DAPI. Each data point shown is mean \pm SEM ( $n=4)$ and is representative of 3 independent experiments 
but whether Smad is necessary for the activation of mTORC1 is not known. Targeted knockdown of Smad3 in pHLFs attenuated mTORC1-dependent phosphorylation of p70S6K and 4E-BP1, indicating that the activation of mTORC1 is Smad3-dependent
(Fig. 4a, b). Smad3 knockdown was also associated with a significant reduction in TGF- $\beta_{1}$-induced COL1A1 mRNA levels and collagen synthesis (Fig. 4c, d). Further support for the conclusion that Smad signaling is temporally upstream of mTOR a

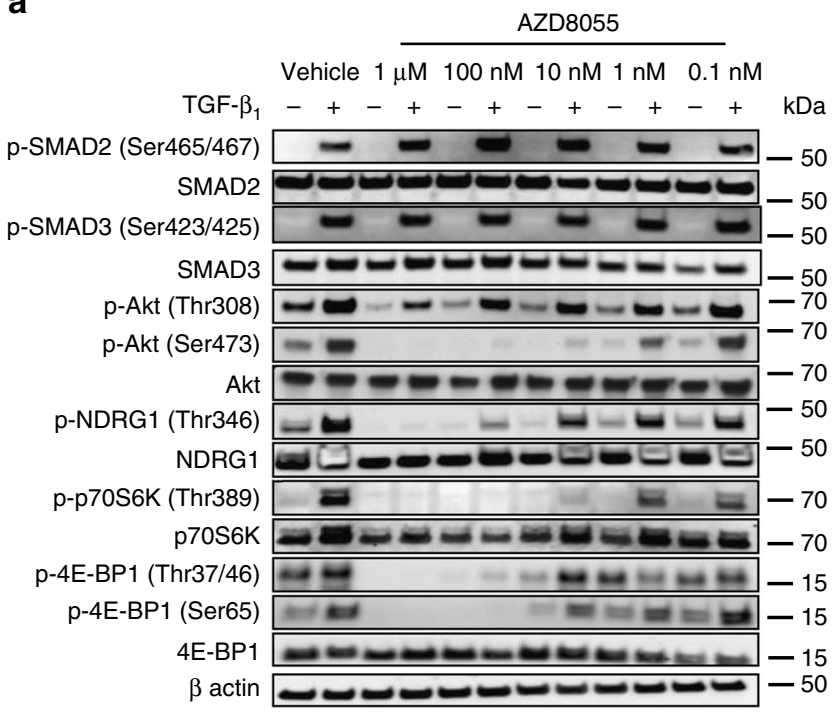

b

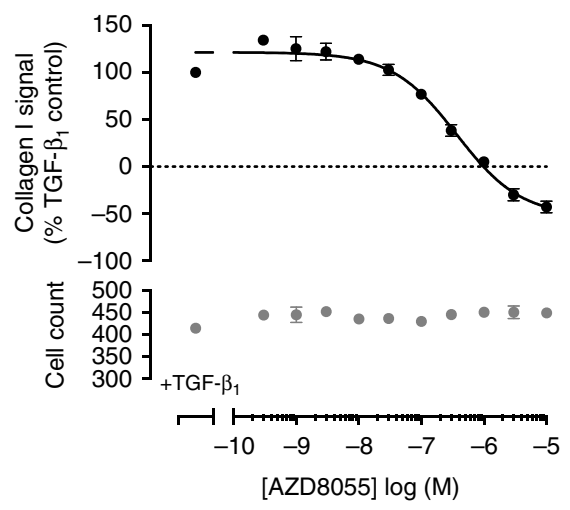

C

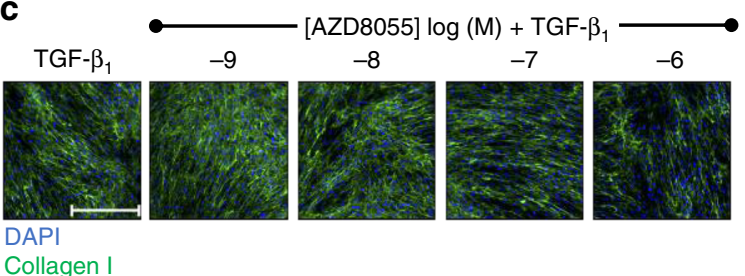

d

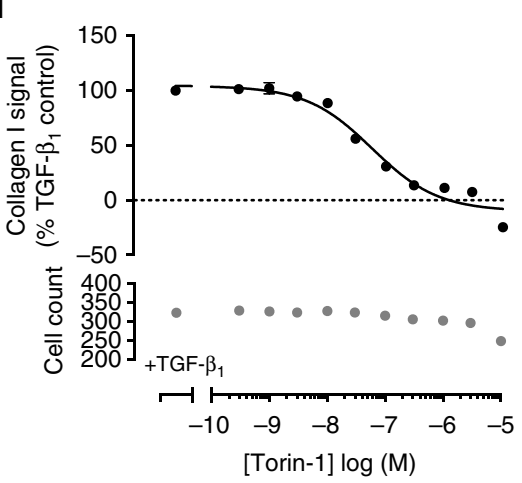

9

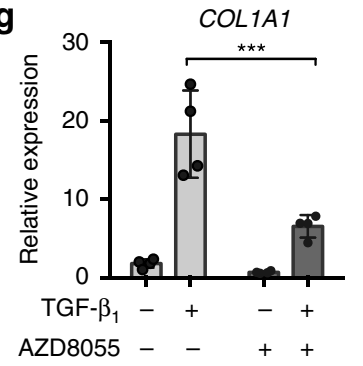

i

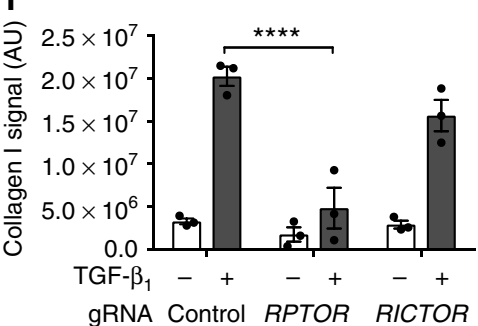

e

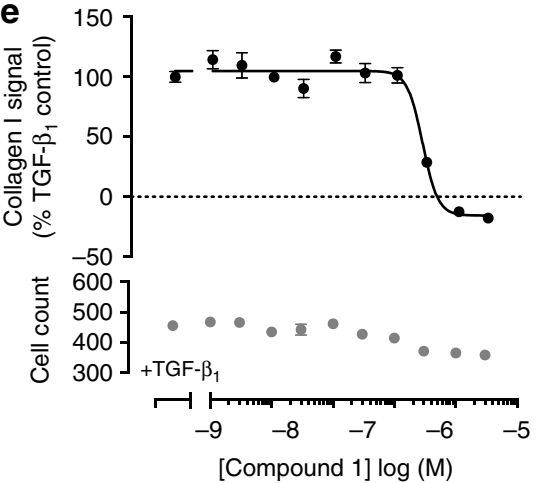

h
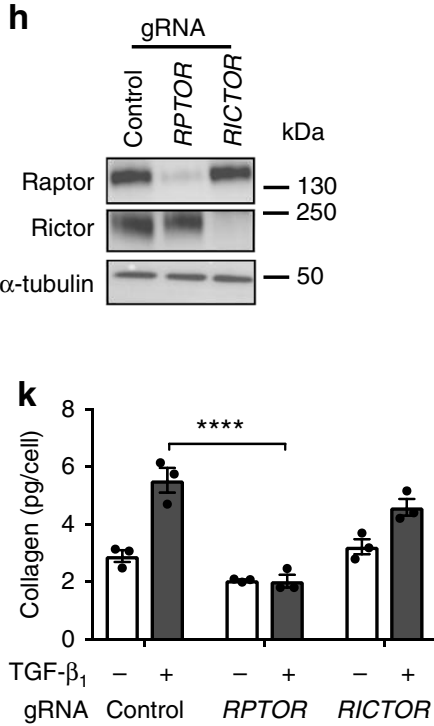

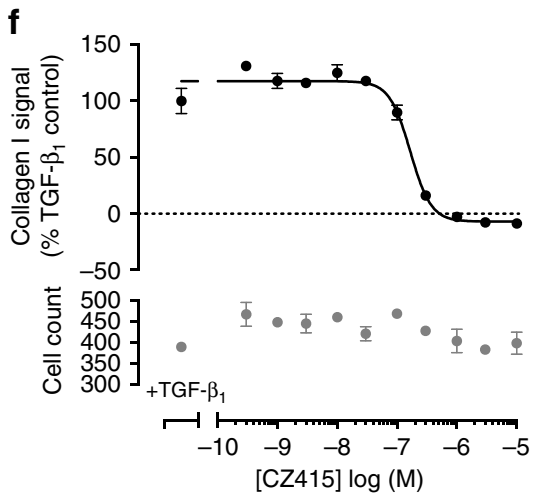

[CZ415] log (M)

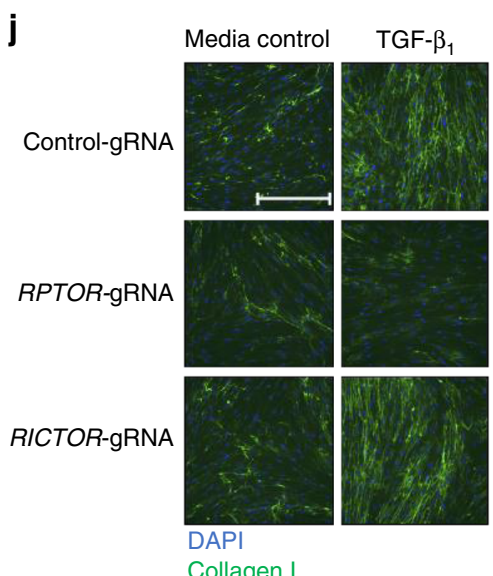


Fig. 3 ATP-competitive inhibition of mTOR and mTORC1 knockout attenuates collagen I deposition in pHLFs. pHLFs were pre-incubated with vehicle (0.1\% DMSO) or increasing concentrations of AZD8055 prior to stimulation with TGF- $\beta_{1}(1 \mathrm{ng} / \mathrm{ml})$. Cells were lysed at $1 \mathrm{~h}$ to assess Smad phosphorylation and at $12 \mathrm{~h}$ to assess the phosphorylation of specified proteins, assessed by Western blot (a). pHLFs were pre-incubated with vehicle (0.1\% DMSO) or increasing concentrations of AZD8055 (b, c), Torin-1 (d), Compound 1 (e), or CZ415 (f) and stimulated with TGF- $\beta_{1}$ for $72 \mathrm{~h}$ with collagen I deposition assessed by macromolecular crowding assay. Data are expressed as collagen I signal calculated as a percentage of the TGF- $\beta_{1}$-treated control ( $n=4$ fields of view imaged per well) and cell counts obtained by staining nuclei with DAPI $(n=4)$. Data are representative of 5 independent experiments. Scale bars $=$ $360 \mu \mathrm{m} . \mathrm{IC}_{50}$ values were calculated using 4-parameter non-linear regression: AZD8055, IC $50=368 \mathrm{nM}, 95 \% \mathrm{Cl} 220-616 \mathrm{nM}$; Torin-1, IC $50=57.8 \mathrm{nM}$, 95\% Cl 38-87.7 nM; Compound 1, IC $\mathrm{I}_{50}=2.6 \mu \mathrm{M}, \mathrm{Cl} 2.1-3.1 \mu \mathrm{M} ; \mathrm{CZ415}, \mathrm{IC} 50=165.9 \mathrm{nM}, 95 \% \mathrm{Cl} 135.4-203 \mathrm{nM}$. COL1A1 mRNA levels were assessed by real-time RT qPCR after pre-incubation of pHLFs with vehicle (0.1\% DMSO) or $1 \mu \mathrm{M}$ AZD8055 prior to TGF- $\beta_{1}$ stimulation for $24 \mathrm{~h}(n=4)(\mathbf{g})$. Relative expression was calculated using $2^{-\Delta C t}$. $\Delta$ Ct was calculated from the geometric mean of two reference genes. pHLFs were modified by CRISPR-Cas9 gene editing using guide RNAs (gRNA) targeting exon 26 of RPTOR or exon 29 of RICTOR. Analysis of the resultant levels of Raptor and Rictor protein are shown (h). CRISPR-Cas9-edited pHLFs were stimulated with TGF- $\beta_{1}(1 \mathrm{ng} / \mathrm{ml})$ for $72 \mathrm{~h}$, with collagen I deposition normalized to cell count assessed by macromolecular crowding assay $(n=3)$ (i). Representative images are shown in $\mathbf{j}$. In addition, supernatants were collected from CRISPR-Cas9-edited pHLFs treated with TGF $-\beta_{1}(1 \mathrm{ng} / \mathrm{ml})$ for $72 \mathrm{~h}$ and hydroxyproline was quantified using HPLC $(n=3)(\mathbf{k})$. Data are presented as means \pm SEM. Differences between groups were evaluated with two-way ANOVA with Tukey multiple comparison testing, ${ }^{\star \star *} p<0.001,{ }^{\star \star \star *} p<0.0001$

a

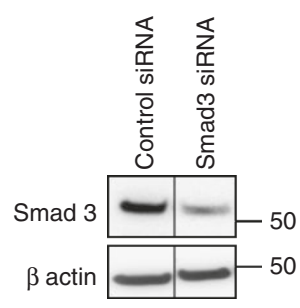

C

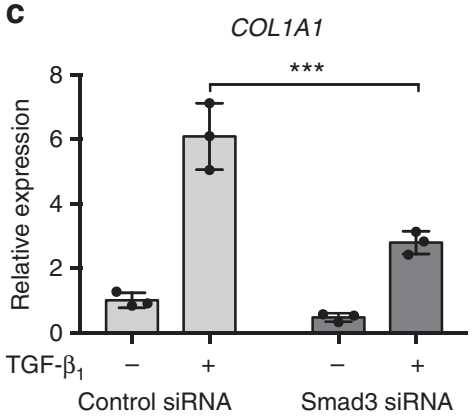

b

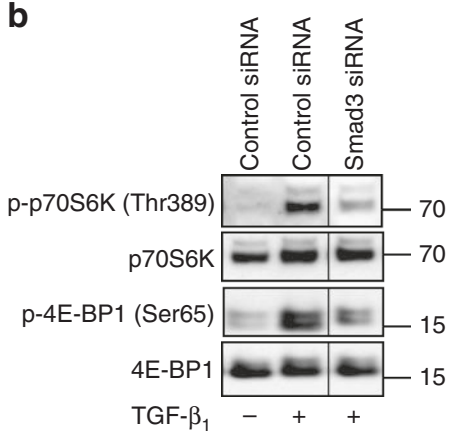

d

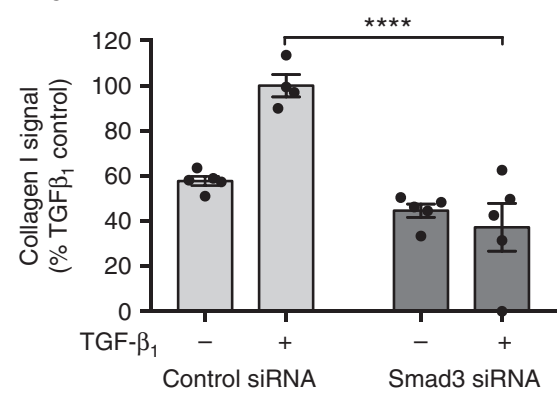

Fig. 4 mTORC1 signaling is Smad-dependent. pHLFs were transfected with control siRNA or siRNA targeting Smad3 and Smad3 protein expression measured by Western blot (a). Following transfection, pHLFs were stimulated with TGF- $\beta_{1}(1 \mathrm{ng} / \mathrm{ml})$ for $12 \mathrm{~h}$ and $\mathrm{mTORC} 1$ signaling evaluated by Western blot analysis (b). An irrelevant lane has been spliced out of the prepared images (a) and (b). Uncropped gels are shown in Supplementary Fig. 9. COL1A1 mRNA levels were assessed by real-time RT qPCR at $24 \mathrm{~h}(n=3)$ (c). Relative expression was calculated using $2^{-\Delta C t}$. $\Delta C t$ was calculated from the geometric mean of two reference genes. Collagen I deposition was measured by macromolecular crowding assay at $72 \mathrm{~h}$ (d). Data are expressed as collagen I signal normalized to cell count ( $n=4$ fields of view imaged per well) calculated as a percentage of the TGF- $\beta_{1}$-treated control $(n=5)$. Data are presented as mean \pm SEM and are representative of 2 independent experiments. Differences between groups were evaluated with two-way ANOVA with Tukey multiple comparison testing, ${ }^{\star \star \star} p<0.001,{ }^{\star \star \star \star} p<0.0001$

was obtained by showing that AZD8055, Torin-1, and Compound 1 have no effect on the phosphorylation of either Smad2 or 3 (Fig. 3a and Supplementary Fig. 3).

TGF- $\beta_{1}$-induced collagen synthesis is rapamycin-insensitive. Having established the importance of mTORC1 signaling for TGF- $\beta_{1}$-induced collagen synthesis, we examined the effect of the partial allosteric mTORC1 inhibitor, rapamycin. In contrast to ATP-competitive mTOR inhibitors, which directly bind to the mTOR catalytic site, rapamycin had no effect on TGF- $\beta_{1}$-induced collagen synthesis (Fig. $5 \mathrm{a}$ ) or the peak increase in COL1A1 mRNA levels (Fig. 5b). Comparison of the effect of AZD8055 versus rapamycin on downstream mTORC1 substrates revealed that treatment with rapamycin completely inhibited p70S6K phosphorylation, whereas its effects on mTORC1-mediated 4EBP1 phosphorylation were modest (Fig. 5c). In contrast, Fig. 3a shows that the ATP-competitive mTORC1/2 inhibitor, AZD8055, completely inhibited phosphorylation of both mTORC1 substrates, p70S6K and 4E-BP1, notably affecting all measured 4E-BP-1 phosphorylation sites.

To further interrogate the role of mTORC1 downstream substrates in TGF- $\beta_{1}$-induced collagen synthesis, we investigated the effects of p70S6K inhibition with LY2584702. This compound $(1 \mu \mathrm{M})$ inhibited the phosphorylation of its downstream substrate, ribosomal protein S6 (Fig. 5d), but had no effect on 
a

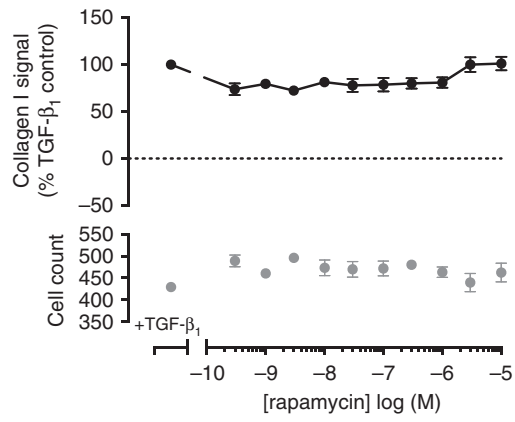

C

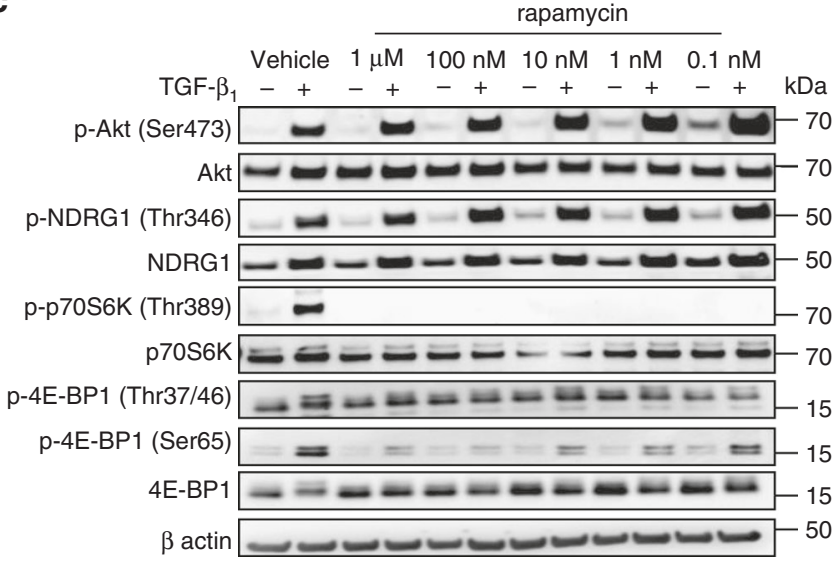

b

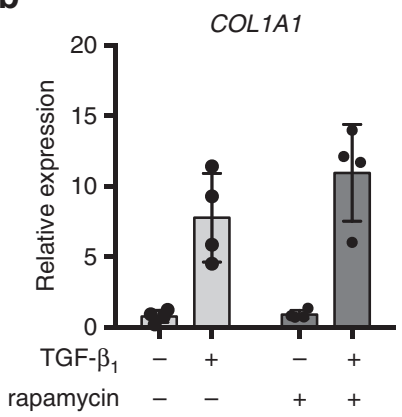

d

TGF- $\beta_{1}-+-+$

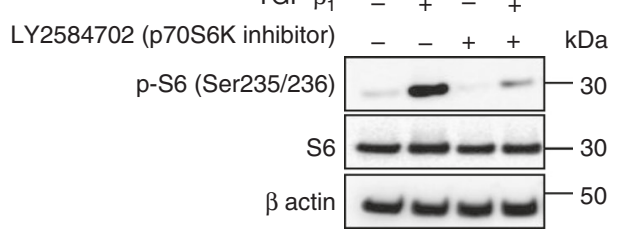

e

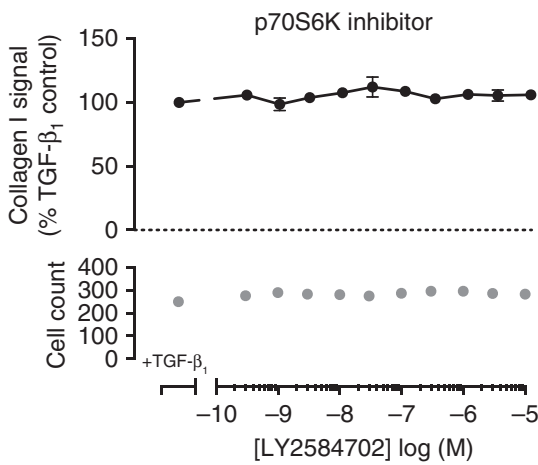

g

f

TGF- $\beta_{1}-+-+$

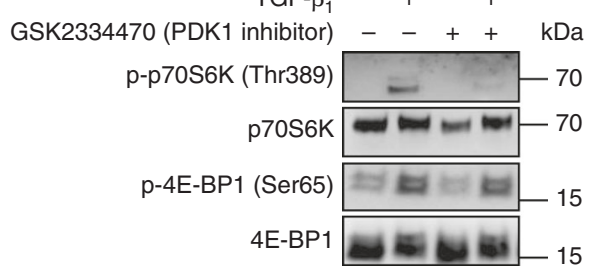

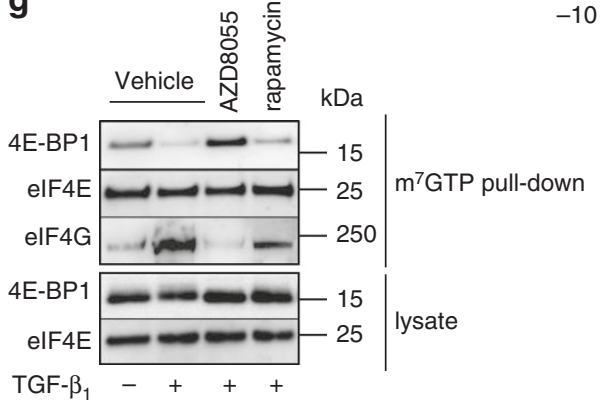

Fig. 5 Rapamycin-insensitive mTORC1 signaling mediates TGF- $\beta_{1}$-induced collagen I deposition. pHLFs were pre-incubated with vehicle (0.1\% DMSO) or increasing concentrations of rapamycin (a) and LY2584702 (e) and stimulated with TGF- $\beta_{1}$ for $72 \mathrm{~h}$ with collagen I deposition assessed by macromolecular crowding assay. Data are expressed as collagen I signal as a percentage of the TGF- $\beta_{1}$-treated control $(n=4$ fields of view imaged per well) and cell counts obtained by staining nuclei with DAPI. Data are presented as mean \pm SEM $(n=4)$ and are representative of 3 independent experiments. Additionally, pHLFs were pre-incubated with vehicle (0.1\% DMSO) or increasing concentrations of rapamycin (c), $1 \mu$ M LY2584702 (d), or $1 \mu$ M GSK2334470 (f) prior to stimulation with TGF- $\beta_{1}(1 \mathrm{ng} / \mathrm{ml})$. Cells were lysed $12 \mathrm{~h}$ later and the phosphorylation of specified proteins was assessed by Western blot. COL1A1 mRNA levels were assessed by RT qPCR after pre-incubation of pHLFs with vehicle (0.1\% DMSO) or 100 nM rapamycin prior to TGF- $\beta_{1}$ stimulation for $24 \mathrm{~h}$ (b). Relative expression was calculated using $2^{-\Delta \mathrm{Ct}} . \Delta \mathrm{Ct}$ was calculated from the geometric mean of two reference genes. Data are presented as mean \pm SEM $(n=4)$. Differences between groups were evaluated with two-way ANOVA. For immunoprecipitation of the $\mathrm{m}^{7} \mathrm{GTP}$ cap, $\mathrm{pHLFs}$ were preincubated with vehicle (0.1\% DMSO), $1 \mu$ M AZD8055 or $100 \mathrm{nM}$ rapamycin prior to TGF- $\beta_{1}$ stimulation. Protein levels were assessed by Western blot (g)

TGF- $\beta_{1}$-induced collagen synthesis (Fig. $5 \mathrm{e}$ ). p70S6K is a member of the AGC kinase superfamily that is sequentially phosphorylated by PDK1 and mTOR to become fully activated. PDK1 inhibition attenuated p70S6K phosphorylation by mTORC1 (Fig. 5f), but as previously mentioned had no effect on TGF- $\beta_{1}$-induced collagen synthesis (Fig. 2f). Taken together, these data led us to conclude that rapamycin-sensitive mTORC1 signaling through $\mathrm{p} 70 \mathrm{~S} 6 \mathrm{~K}$ is dispensable for TGF- $\beta_{1}$-induced collagen synthesis.
The role of 4E-BP1 downstream of mTORC1 signaling. Having ruled out p70S6K, we next addressed the potential involvement of 4E-BP1. 4E-BP1 is a major translational repressor which inhibits cap-dependent translation by binding to eukaryotic translation initiation factor (eIF)4E, preventing the recruitment of eIF4G and the subsequent formation of the translation initiation complex. Dissociation of 4E-BP1 from eIF4E is dependent on the stepwise phosphorylation of at least four critical sites by mTORC1 
a

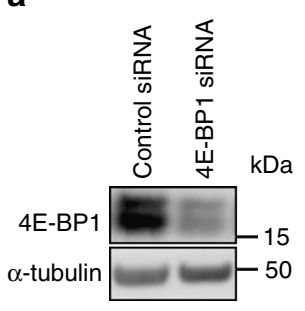

b

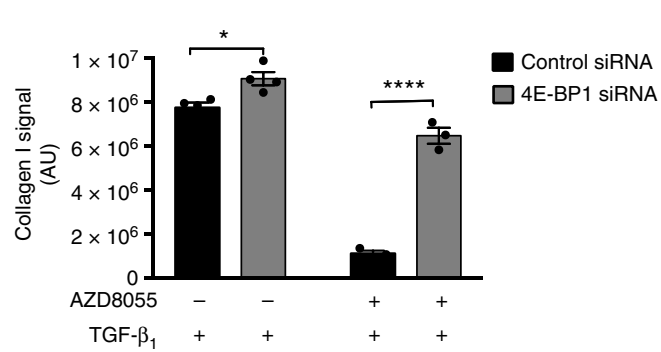

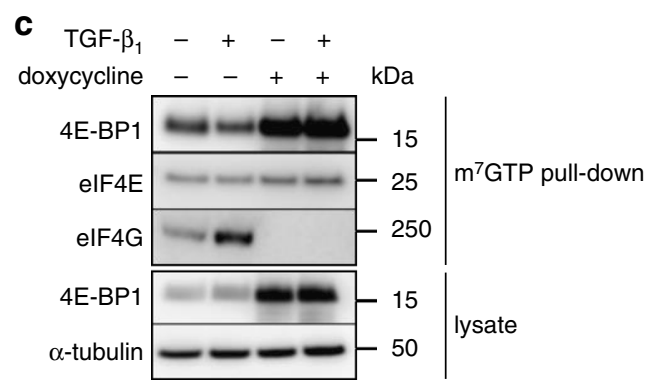

d

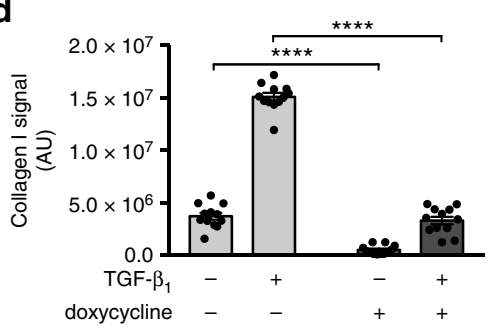

f

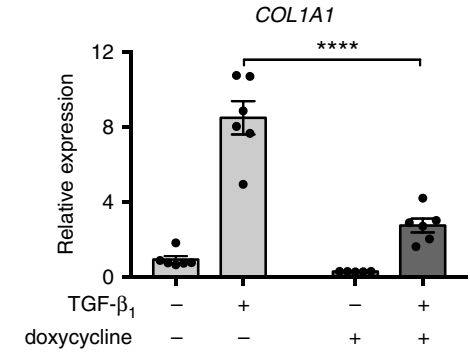

g

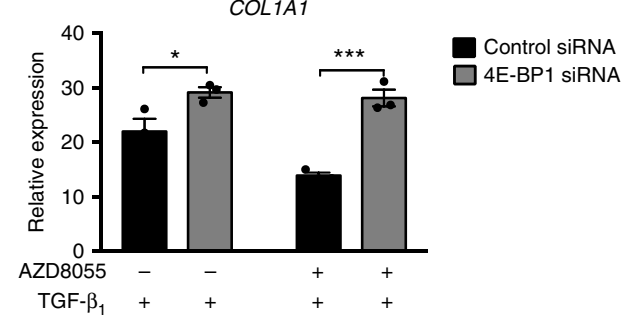

e
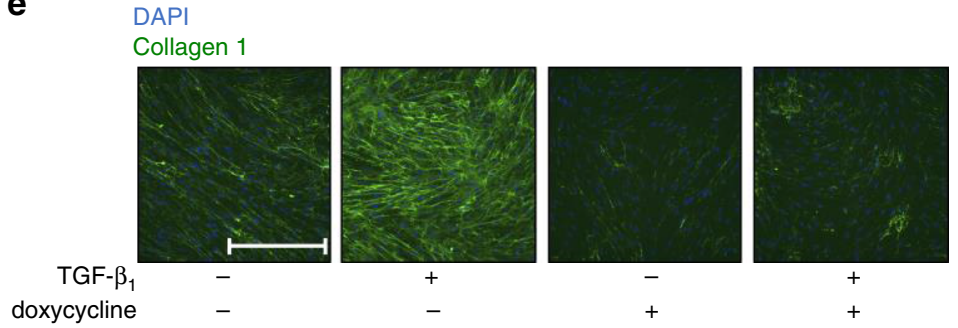

h

h TGF- $\beta$

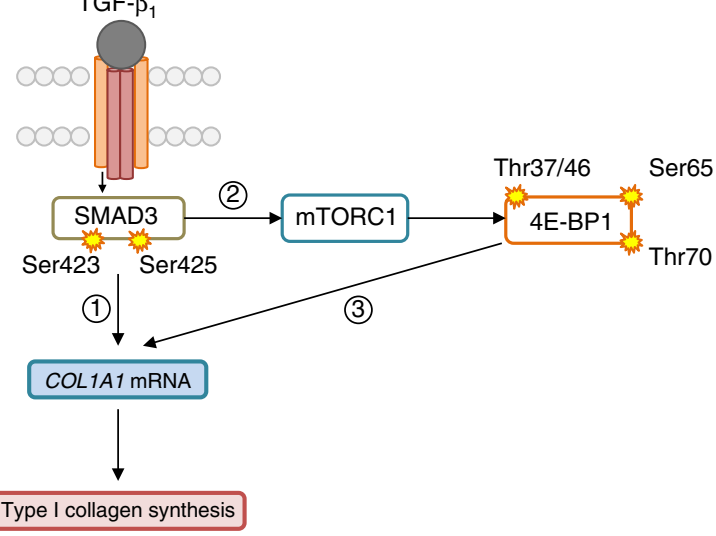

Fig. 6 mTORC1/4E-BP1 axis mediates the TGF- $\beta_{1}$ collagen I response in pHLFs. pHLFs were transfected with control siRNA or siRNA targeting 4E-BP1. 4EBP1 protein expression was measured by Western blot (a). Following transfection, pHLFs were preincubated with $1 \mu$ M AZD 8055 or vehicle (0.1\% DMSO) prior to stimulation with TGF- $\beta_{1}(1 \mathrm{ng} / \mathrm{ml})$ for $72 \mathrm{~h}$. Collagen I deposition was assessed by macromolecular crowding assay ( $\left.n=4\right)(\mathbf{b})$. COL1A1 mRNA levels at $24 \mathrm{~h}$ were assessed by real-time RT qPCR $(n=3)(\mathrm{g})$. pHLFs expressing a 4E-BP1-4A dominant-negative phosphomutant were treated with doxycycline $(1 \mu \mathrm{g} / \mathrm{ml})$ or media control for $24 \mathrm{~h}$ prior to TGF- $\beta_{1}$ stimulation. Immunoprecipitation of the $\mathrm{m}^{7} \mathrm{GTP}$ cap was performed and protein levels assessed by Western blot (c). Collagen deposition was measured at $72 \mathrm{~h}$ by macromolecular crowding assay $(n=12)(\mathbf{d})$. Representative images are shown in e. Scale bars $=360 \mu \mathrm{m}$. COL1A1 mRNA levels were measured at $24 \mathrm{~h}(n=6)(\mathbf{f})$. Data are presented as means \pm SEM. For real-time RT-PCR, relative expression was calculated using $2^{-\Delta C t} . \Delta \mathrm{Ct}$ was calculated from the geometric mean of two reference genes. For collagen deposition assays all data are expressed as collagen I signal ( $n=4$ fields of view imaged per well) normalized to cell count. All data are representative of at least 3 independent experiments. Differences between groups were evaluated with two-way ANOVA with Tukey multiple comparison testing, ${ }^{\star} p<0.05,{ }^{\star \star \star *} p<0.001,{ }^{\star \star \star \star} p<$ 0.0001. Proposed model is shown in $\mathbf{h}$ (schematic generated by author J.D.E.). TGF- $\beta_{1}$ activates the Smad3 pathway which in turn directly influences early increases in COL1A1 mRNA levels (1), as has been previously described. Smad3 activation subsequently activates the mTORC1/4E-BP1 axis (2). This cascade regulates maximal COLTA1 mRNA levels through an unknown mechanism, likely involving the translational regulation of a protein intermediate (3)

(threonine residues 37,46 , and 70 and serine residue 65 ) and allows eIF4G to bind eIF4E to facilitate cap-dependent translation $^{28,29}$. Cap pull-down studies with the $\mathrm{m}^{7} \mathrm{GTP}$ cap analog with lysates from TGF- $\beta_{1}$-treated fibroblasts in the presence of either rapamycin or AZD8055 revealed that TGF- $\beta_{1}$ stimulation led to the release of $4 \mathrm{E}-\mathrm{BP} 1$, allowing eIF4G to bind to the cap complex (Fig. 5g). Treatment with AZD8055 enhanced the binding of $4 \mathrm{E}-\mathrm{BP} 1$ to the cap complex, excluding eIF4G despite TGF- $\beta_{1}$ stimulation. In contrast, rapamycin treatment failed to prevent
4E-BP1 dissociation from the cap complex in TGF- $\beta_{1}$-stimulated fibroblasts. Taken together, these data suggest that only ATPcompetitive mTOR inhibition, which fully suppresses 4E-BP1 phosphorylation downstream of mTORC1 to enhance 4E-BP1 binding to the cap (Fig. 3a and Supplementary Fig. 3), is capable of preventing the initiation of TGF- $\beta_{1}$-induced cap-dependent translation.

To further interrogate the role of $4 \mathrm{E}-\mathrm{BP} 1$, we knocked down $4 \mathrm{E}-\mathrm{BP} 1$ by targeted siRNA (which should mimic full 4E-BP1 

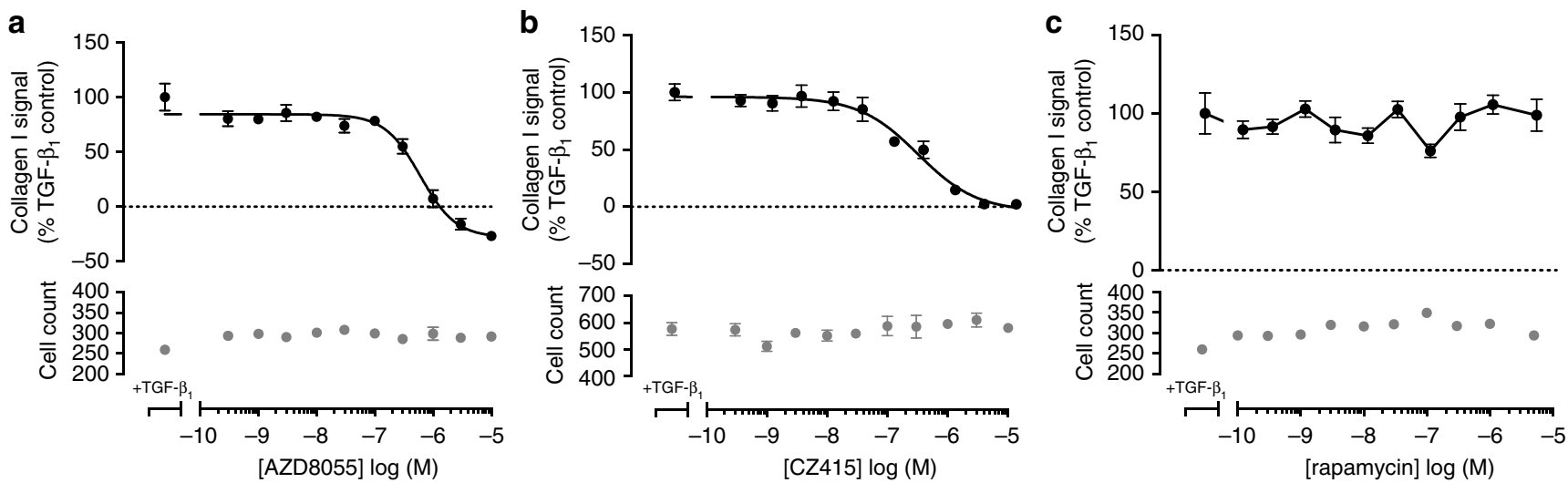

d
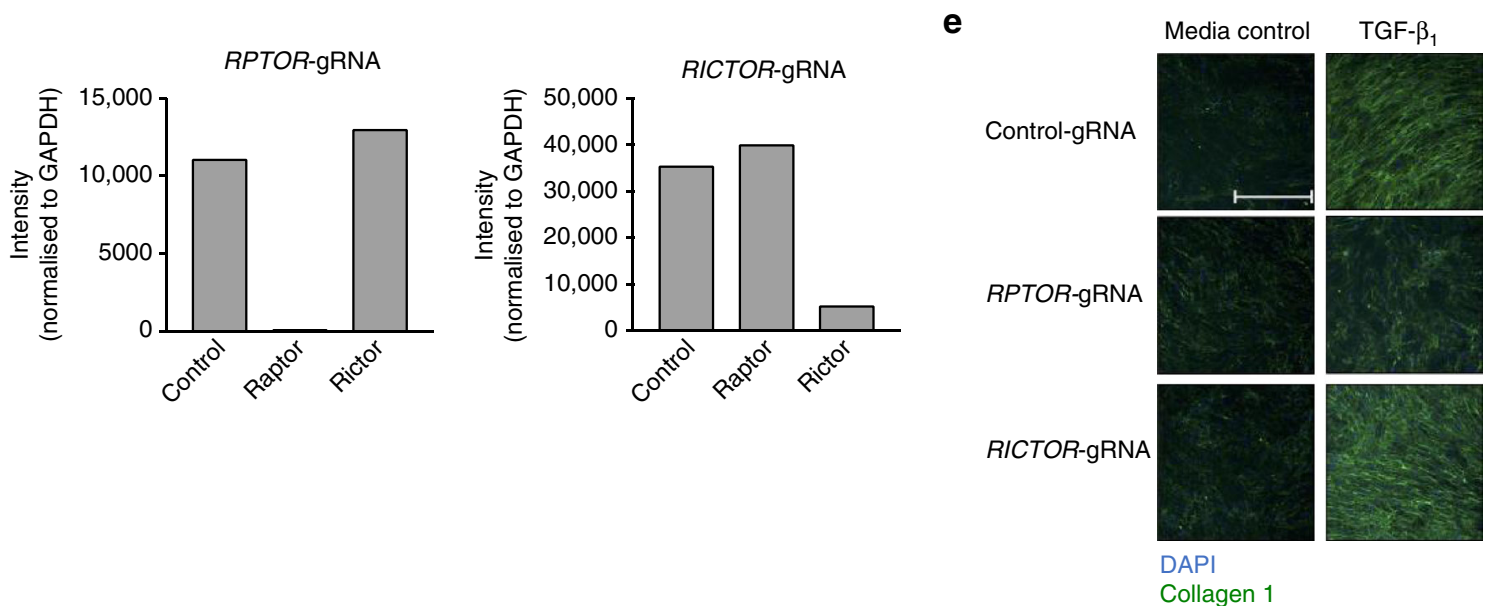

Fig. 7 mTORC1 plays a critical role in mediating the pro-fibrotic effects of TGF- $\beta_{1}$ in IPF-derived lung fibroblasts. IPF human lung fibroblasts (IPF-HLFs) were pre-incubated with vehicle (0.1\% DMSO) or increasing concentrations of AZD8055 (a), CZ415 (b), or rapamycin (c) and stimulated with TGF- $\beta_{1}(1 \mathrm{ng} / \mathrm{ml})$ for $72 \mathrm{~h}$ with collagen I deposition assessed by macromolecular crowding assay. Data are expressed as collagen I signal calculated as a percentage of the TGF- $\beta_{1}$-treated control ( $n=4$ fields of view imaged per well) and cell counts obtained by staining nuclei with DAPI. Each data point shown is mean \pm SEM $(n=4)$ and is representative of 5 independent experiments. IC $C_{50}$ values were calculated using 4-parameter non-linear regression: AZD8055, IC ${ }_{50}=$ $604 \mathrm{nM}, 95 \% \mathrm{Cl} 415 \mathrm{nM}$ to $1 \mu \mathrm{M} ; \mathrm{CZ415}, \mathrm{IC}_{50}=245.3 \mathrm{nM}, 95 \% \mathrm{Cl} 136.7-541 \mathrm{nM}$. Additionally, IPF-HLFs were modified by CRISPR-Cas9 gene editing using gRNAs targeting exon 26 of RPTOR or exon 29 of RICTOR. Analysis of the resultant levels of Raptor and Rictor protein are shown (d). CRISPR-Cas9edited IPF-HLFs were also stimulated with TGF- $\beta_{1}(1 \mathrm{ng} / \mathrm{ml})$ for $72 \mathrm{~h}$, with collagen I deposition assessed by macromolecular crowding assay (e). Scale bars $=360 \mu \mathrm{m}$

phosphorylation). This led to an enhanced TGF- $\beta_{1}$ response and interfered with the ability of AZD8055 to inhibit TGF- $\beta_{1}$ induced collagen synthesis (Fig. 6a, b) indicating that the inhibitory effects of AZD8055 are mediated by blocking the phosphorylation of $4 \mathrm{E}$ BP1. To further interrogate the mTORC1/4E-BP1 axis, we generated pHLFs expressing a doxycycline-inducible 4E-BP1 dominant negative phospho-mutant (with mTORC1 phosphorylation sites Thr37, Thr46, Ser65, and Thr70 replaced by alanine, abbreviated as $\left.4 \mathrm{E}-\mathrm{BP} 1-4 \mathrm{~A}^{29}\right)$. We reasoned that the expression of $4 \mathrm{E}-\mathrm{BP} 1-4 \mathrm{~A}$ would mimic the effects of ATP-competitive mTOR inhibition on collagen deposition. $\mathrm{m}^{7} \mathrm{GTP}$ cap pull-down confirmed doxycycline treatment induced 4E-BP1-4A expression and constitutive binding of $4 \mathrm{E}-\mathrm{BP} 1-4 \mathrm{~A}$ to eIF4E (Fig. 6c). Doxycycline had no effect on 4E-BP1 expression or collagen deposition in untransduced pHLFs (Supplementary Fig. 5a, b). Expression of the dominant negative 4E-BP1-4A phosphomutant resulted in a marked attenuation of TGF- $\beta_{1}$-induced collagen deposition to below constitutive baseline values (Fig. 6d, e).

4E-BP1-4A expression also inhibited TGF- $\beta_{1}$-induced COL1A1 mRNA levels (Fig. 6f); whereas 4E-BP1 siRNA knockdown similarly rescued the inhibitory effect of AZD8055 on TGF- $\beta_{1^{-}}$ induced COL1A1 mRNA levels (Fig. 6g), indicating that the mTORC1/4E-BP1 axis acts at least in part, at the level of regulating COL1A1 mRNA levels to mediate the fibrogenic effects of TGF- $\beta_{1}$ in pHLFs. Our proposed model is shown in Fig. $6 \mathrm{~h}$.

mTORC1 signaling and fibrogenesis in IPF. All studies thus far were performed with pHLFs from control donors. Since IPF fibroblasts have been reported to be epigenetically modified (reviewed in ref. ${ }^{8}$ ), we next examined the role of mTOR signaling in IPF-derived fibroblasts. ATP-competitive mTOR inhibition with AZD8055 (Fig. 7a) and CZ415 (Fig. 7b) was also found to inhibit TGF- $\beta_{1}$-induced collagen synthesis in a concentrationdependent manner in IPF fibroblasts. This observation was confirmed in 4 additional IPF donor lines (Supplementary Table 3). As observed for control fibroblasts, the TGF- $\beta_{1}$ collagen response was completely rapamycin-insensitive in IPF fibroblasts (Fig. 7c).

We were also successful in performing CRISPR-Cas9 gene editing of RPTOR (mTORC1) or RICTOR (mTORC2) in IPF fibroblasts as evidenced by marked reduction in Raptor and Rictor protein expression (Fig. 7d). As observed for control fibroblasts, TGF- $\beta_{1}$-induced collagen deposition was maintained in RICTOR-silenced cells but RPTOR gene editing resulted in near-complete inhibition of TGF- $\beta_{1}$-induced collagen synthesis 
a
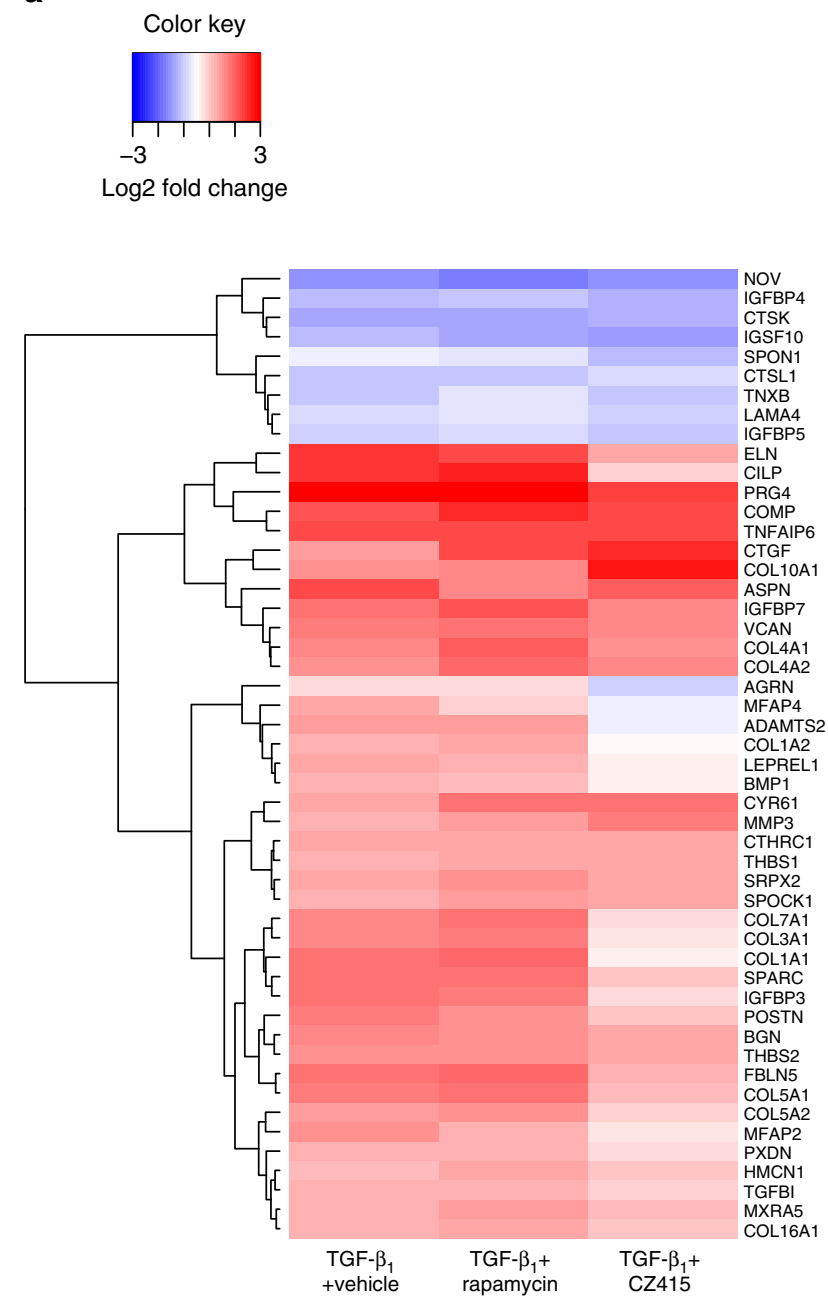

b

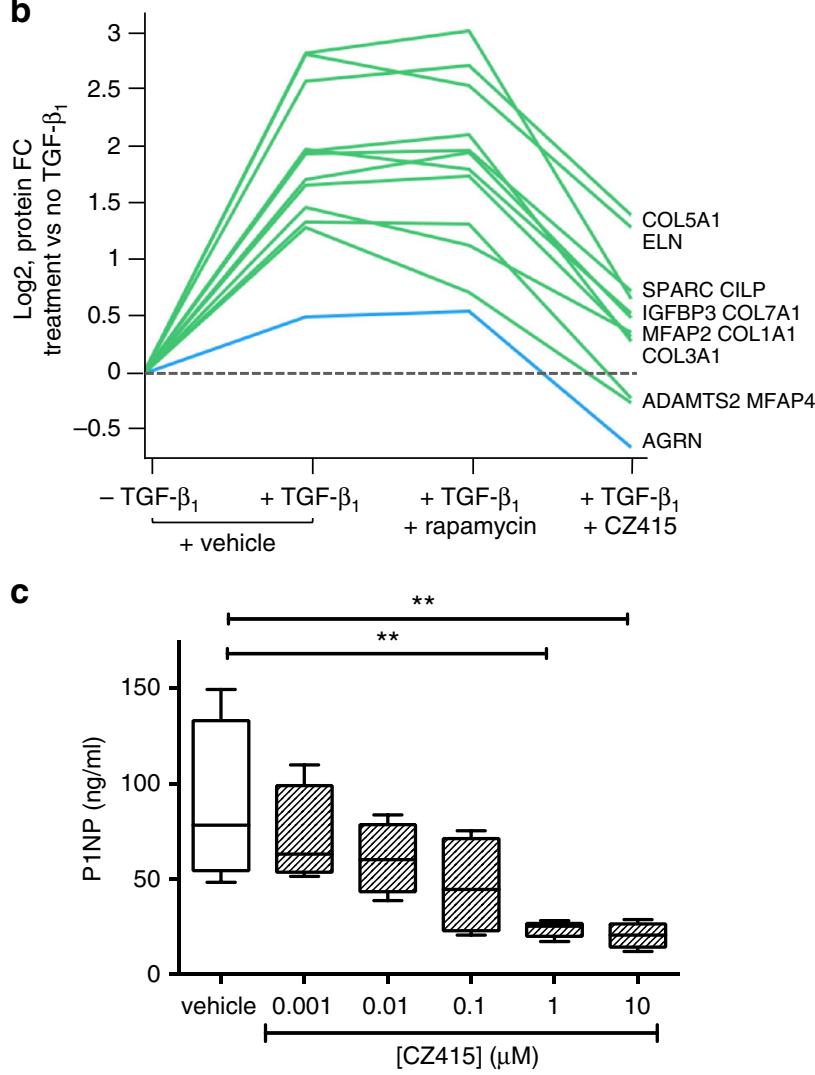

d

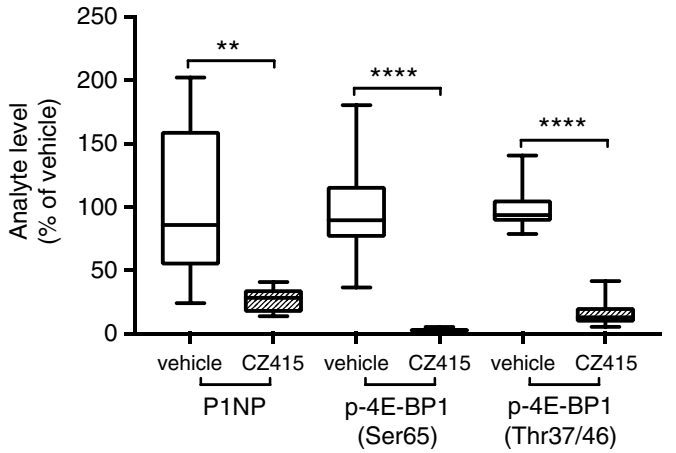

Fig. 8 ATP-competitive mTOR inhibition attenuates TGF- $\beta_{1}$-induced matrisome protein expression in vitro and collagen synthesis in IPF lung slices. IPF human lung fibroblasts (IPF-HLFs) were pre-incubated with vehicle (0.1\% DMSO), rapamycin (100 $\mathrm{nM}$ ) or the mTOR inhibitor CZ415 (5 $\mu \mathrm{M})$ prior to stimulation with TGF $-\beta_{1}(1 \mathrm{ng} / \mathrm{ml})$ for $24 \mathrm{~h}$. Proteomic analysis representing fold change (FC) of core matrisome proteins and proteins involved in collagen synthesis and degradation in lysates of TGF- $\beta_{1}+$ vehicle/drug-treated fibroblasts versus negative TGF- $\beta_{1}$ control is displayed in a heatmap. Only proteins with up- or down-regulation ( $p$ Adj $<0.05$ and $|F C|>1.2$ for both replicates) in any treatment versus negative TGF- $\beta_{1}$ control are represented (50 proteins) (a). Quantitative profiles across treatments are displayed as line charts (b). Human IPF lung tissue slices generated from IPF lung transplant tissue were treated with vehicle (0.1\% DMSO) or increasing concentrations of CZ415 for $120 \mathrm{~h}$. Levels of P1NP in supernatants from 4 slices per condition were assessed by ELISA (c). Data are representative of 5 independent donors. Differences between conditions were evaluated with one-way ANOVA with Dunnett's multiple comparison testing, ${ }^{\star \star} p<0.005$. Human IPF lung tissue slices $(n=4)$ from 2 donors were treated with vehicle $(0.1 \%$ DMSO) or CZ415 $(1 \mu \mathrm{M})$ for 5 days and P1NP levels in supernatants and phosphoproteins in homogenized slices were measured. Pooled donor data are presented in d. Differences between conditions were evaluated with Student's $t$-test, ${ }^{\star \star} p<0.005,{ }^{\star \star \star \star} p<0.0001$. In $\mathbf{c}$, $\mathbf{d}$ the boxplot center line, bounds of box, and whiskers represent median, inter-quartile range, and minimum to maximum values

(Fig. 7e) indicating that mTORC1 is also a key signaling node during fibrogenesis in IPF fibroblasts.

We next explored the potential role of mTOR in the broader context of regulating the TGF- $\beta_{1}$-modulated matrisome by conducting a proteomic analysis of IPF fibroblasts grown under identical macromolecular crowding conditions in the presence of either CZ415 or rapamycin (Fig. 8a). We focus here on the core matrisome proteins (http://matrisomeproject.mit.edu/ other-resources/human-matrisome/) and proteins involved in collagen synthesis and degradation (https://reactome.org/ PathwayBrowser/\#/R-HSA-1650814); the complete data is in Supplementary Data 2. Among these matrisome proteins, about $50 \%(169 / 330)$ were detectable in our cell system. Figure 8a represents a heatmap of the 50 proteins that showed a fold change (FC) ( $p \mathrm{Adj}<0.05$ and $|\mathrm{FC}|>1.2$ in both replicates) in any one condition relative to the negative TGF- $\beta_{1}$ control. Among these, 41 proteins showed increased levels and 9 showed decreased levels after $24 \mathrm{~h}$ stimulation with TGF- $\beta_{1}$. To identify differences 
a

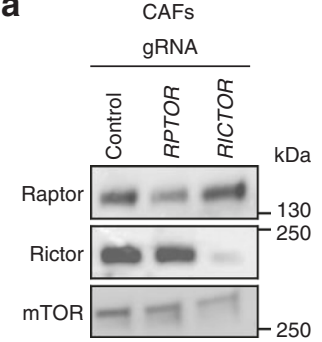

e

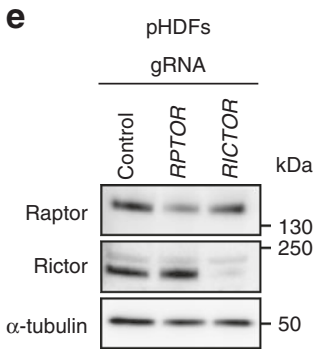

i

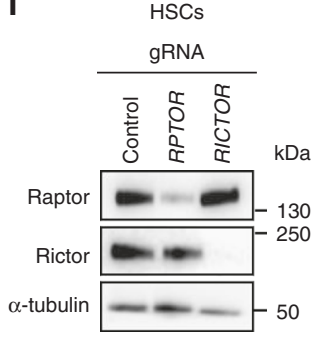

b

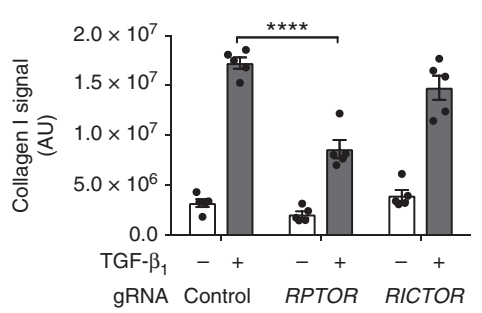

f

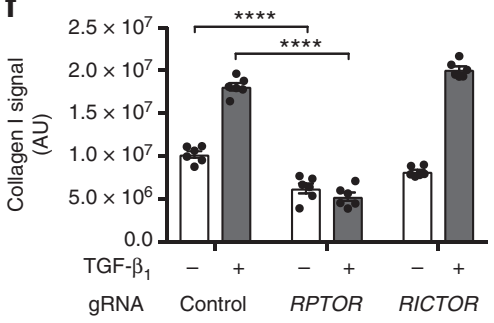

j

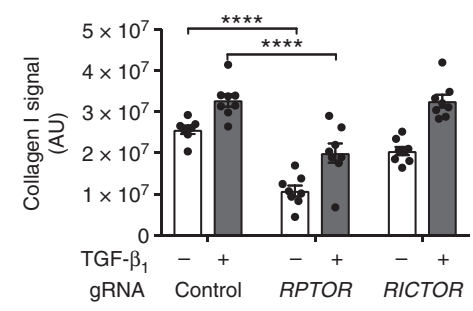

C

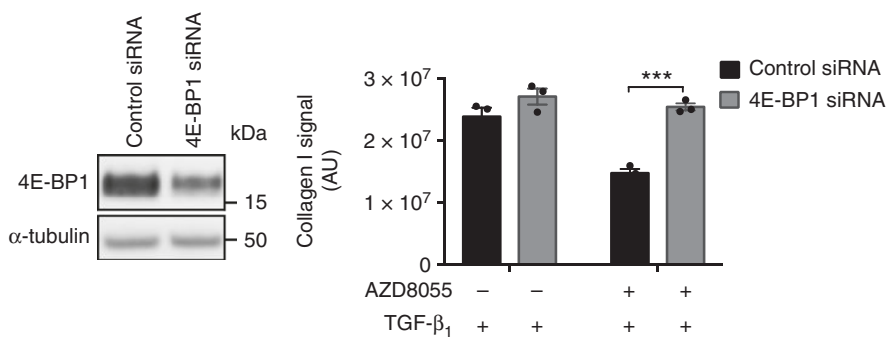

g

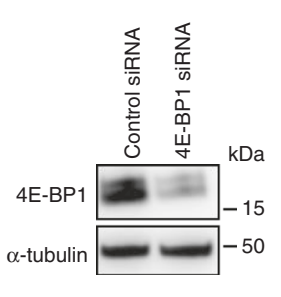

h

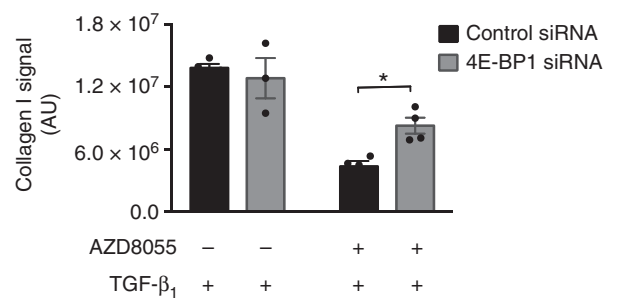

$\mathbf{k}$

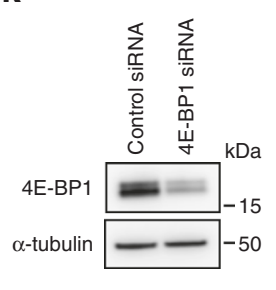

I

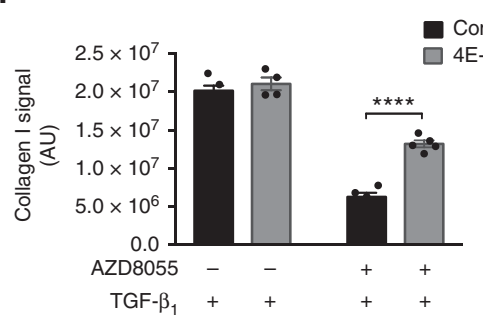

Fig. 9 mTORC1/4E-BP1 axis mediates collagen I deposition in other mesenchymal cells. Lung adenocarcinoma-associated fibroblasts (CAFs), primary human dermal fibroblasts (pHDFs), and primary hepatic stellate cells (HSCs) were modified by CRISPR-Cas9 gene editing using gRNAs targeting exon 26 of RPTOR or exon 29 of RICTOR. Analysis of the resultant levels of Raptor and Rictor protein are shown (a, e, i). CRISPR-Cas9-edited CAFs, pHDFs, and HSCs were stimulated with TGF- $\beta_{1}(1 \mathrm{ng} / \mathrm{ml})$ for $72 \mathrm{~h}$, with collagen I deposition assessed by macromolecular crowding assay (b, $\left.\mathbf{f}, \mathbf{j}\right)$. Data are expressed as collagen intensity ( $n=4$ fields of view imaged per well) normalized to cell count. Data are presented as mean \pm SEM (CAFs $n=5$, pHDFs $n=6$, HSCs $n=8)$. CAFs, pHDFs, and HSCs were transfected with control siRNA or siRNA targeting 4E-BP and 4E-BP1 protein expression was measured (c, $\mathbf{g}$, $\mathbf{k}$ ). Following transfection, cells were preincubated with $1 \mu \mathrm{M}$ (CAFs) or $300 \mathrm{nM}$ (pHDFs, HSCs) AZD8055 or vehicle prior to stimulation with TGF- $\beta_{1}$ for $72 \mathrm{~h}$. Collagen I deposition was analyzed by macromolecular crowding assay $(\mathbf{d}, \mathbf{h}, \mathbf{I})$. Data are expressed as collagen intensity $(n=4$ fields of view imaged per well) normalized to cell count. Data are presented as mean \pm SEM (CAFs $n=3$, pHDFs $n=4, \mathrm{HSCs} n=5$ ). Differences between groups were evaluated with two-way ANOVA with Tukey multiple comparison testing, ${ }^{\star} p<0.05,{ }^{\star \star \star} p<0.001,{ }^{\star \star \star \star} p<0.0001$

between CZ415 and rapamycin on the TGF- $\beta_{1}$ response, we selected proteins that were upregulated by TGF- $\beta_{1}$, inhibited by CZ415, and insensitive to rapamycin treatment as depicted in Fig. 8b. This group included several collagen types (I, III, V, VII), as well as other ECM proteins (e.g., elastin/ELN) and matricellular proteins (e.g., SPARC) and the key procollagen processing enzyme, ADAMTS2 (also known as procollagen I N-proteinase). Of note, the procollagen processing enzyme BMP1 (responsible for cleaving the C-propeptide of fibrillar collagens) presented the same profile but just missed the significance cut-off for one replicate $(p$ Adj $=0.051)$. Finally, the proteoglycan Agrin (AGRN) was not affected by TGF- $\beta_{1}$ or rapamycin, but was significantly down-regulated by CZ415 relative to both TGF- $\beta_{1}$ treatment and vehicle control levels.

In order to determine if there is a functional link between mTOR signaling and collagen synthesis in the context of human fibrotic lung disease, we next evaluated the effect of the most potent and selective ATP-competitive mTOR inhibitor, CZ415, in live unmanipulated precision-cut lung slices generated from IPF lung transplant tissue. De novo collagen synthesis was monitored by measuring the release of the human procollagen 1 formation marker; amino-terminal peptide (P1NP) by proprietary ELISA
(Nordic Bioscience). Figure $8 \mathrm{c}$ shows that P1NP levels in slice supernatants were reduced in the presence of CZ415 in a concentration-dependent manner. This observation was confirmed in tissue slices obtained from 5 additional IPF donors (Supplementary Table 5). To confirm target engagement of the mTORC1/4E-BP1 axis, 4E-BP1 phosphorylation was measured in tissue slice homogenates following exposure to CZ415 $(1 \mu \mathrm{M})$. Inhibition of supernatant P1NP levels was associated with significant attenuation of 4E-BP1 Ser65 and Thr37/46 phosphorylation (Fig. 8d).

mTORC1/4E-BP1 is a core fibrogenic pathway. We also examined the relative contribution of mTORC1 and mTORC2 signaling in primary human dermal fibroblasts (pHDFs), hepatic stellate cells (HSCs), and cancer-associated fibroblasts (CAFs) derived from patients with lung adenocarcinoma by CRISPR-Cas9 gene editing to disrupt mTORC1 (RPTOR) and mTORC2 (RICTOR) signaling. These studies revealed that the magnitude of the TGF- $\beta_{1}$ collagen response was variable and appeared to be related to higher baseline levels of collagen I deposition observed, especially for HSCs. Moreover, 
CAFs displayed evidence of heterogeneous phenotypes as has been extensively reported ${ }^{30}$. For the purpose of this study, we focused on collagen-producing CAFs. Successful gene editing was confirmed at the protein level for all lines tested (Fig. 9a, e, i). Importantly, all cell types in which RPTOR was disrupted were unable to mount a full TGF- $\beta_{1}$ induced collagen response. In contrast, this response was fully maintained in cells in which RICTOR was disrupted (Fig. 9b, $\mathrm{f}, \mathrm{j}$ ). We also determined whether 4E-BP1 siRNA was able to restore the attenuated TGF- $\beta_{1}$ induced collagen response in cells treated with AZD8055. 4E-BP1 was successfully knocked down at the protein level by targeted siRNA (Fig. 9c, g, k). In all cell types examined, 4E-BP1 siRNA rescued the impact of AZD8055 on TGF- $\beta_{1}$ induced collagen synthesis (Fig. 9d, h, l), suggesting that the mTORC1/4E-BP1 axis represents a common fibrogenic signaling hub across stromal cells derived from different organs.

\section{Discussion}

Highly synthetic myofibroblasts are the key effector cells involved in excessive ECM deposition in multiple fibrotic conditions, including IPF. We recently reported that combined pan-PI3K and mTOR inhibition with Omipalisib attenuates TGF- $\beta_{1}$-induced collagen synthesis in human lung fibroblasts (HLFs) ${ }^{22}$. We now dissect the relative contributions of PI3K and mTOR signaling to TGF- $\beta_{1}$-induced collagen deposition and report that rapamycininsensitive mTORC1/4E-BP1 signaling is critical for this response in control and IPF-derived fibroblasts, whereas upstream canonical PI3K/Akt signaling is dispensable. Moreover, the effects of mTOR inhibition extend to other matrisome proteins implicated in the fibrotic response. Targeting mTOR by ATP-competitive mTOR inhibition further blocks collagen synthesis in live IPF tissue slices. Finally, the mTORC1/4E-BP1 axis is also critical for collagen I synthesis by dermal fibroblasts, HSCs, and lung cancerassociated fibroblasts.

PI3K/Akt signaling in response to TGF- $\beta_{1}$ stimulation in HLFs has previously been implicated in pro-survival and anti-apoptotic signaling ${ }^{31,32}$. We now provide strong pharmacological evidence that this axis is however not required for TGF- $\beta_{1}$-induced collagen synthesis in that class 1 PI3K, Akt, and PDK1 inhibitors, which robustly attenuated Akt signaling, consistently had no effect on TGF- $\beta_{1}$-induced collagen deposition in our assays. Although this observation contrasts with previous studies reporting a role for PI3K in TGF- $\beta_{1}$ mediated collagen synthesis $^{33-35}$, these earlier studies employed first generation PI3K inhibitors, LY294002 and wortmannin, which inhibit a broad range of other $\mathrm{PI} 3 \mathrm{~K}$-related proteins, including $\mathrm{mTOR}^{36}$. In contrast, Compound 2 used here exhibits exquisite selectivity for all class $1 \mathrm{PI} 3 \mathrm{~K}$ isoforms over mTOR.

Whilst the PI3K/Akt axis is one of the best-characterized pathways leading to mTORC1 activation ${ }^{37}$, PI3K and Akt inhibition did not impact TGF- $\beta_{1}$-induced mTORC1 downstream signaling. This is consistent with the temporal kinetics of the signaling response to TGF- $\beta_{1}$, in that maximal phosphorylation of mTORC1 substrates was consistently observed at least $10 \mathrm{~h}$ before maximal phosphorylation of Akt. We considered the potential involvement of a number of potential PI3K/Akt independent pathways that could regulate $\mathrm{mTORC1}$ activation in response to TGF- $\beta_{1}$ stimulation. The TSC complex is a key control switch for mTORC1 activation and is phosphorylated by multiple other inputs besides Akt, including MAPK, Winglessrelated integration (Wnt) signaling, redox and nutrient status ${ }^{19}$. Our data show that, while MAPK-dependent TSC2 phosphorylation occurs in response to TGF- $\beta_{1}$, pharmacological inhibition of the major MAPKs implicated in TSC phosphorylation, ERK or RSK1, did not inhibit the downstream TGF- $\beta_{1}$ collagen response.
Furthermore, we ruled out the possibility of compensation between the Akt and ERK pathways, as reported in other cell contexts $^{38}$, on the basis of a lack of an inhibitory effect observed by combined inhibition of Akt and ERK.

In contrast to the lack of evidence supporting the involvement of PI3K and Akt and MAPK signaling, ATP-competitive mTOR inhibition had a profound inhibitory effect on TGF- $\beta_{1}$-induced collagen synthesis (with the most potent and selective compounds tested displaying an $\mathrm{IC}_{50}$ within the nanomolar range) in control and IPF fibroblasts. We subsequently established a critical role for rapamycin-insensitive $\mathrm{mTORC} 1$ signaling in mediating the fibrogenic responses of TGF- $\beta_{1}$ on the basis of evidence obtained by CRISPR-Cas9 gene-editing of RPTOR and the lack of effect of rapamycin treatment on this response, as has been reported by others $^{39,40}$.

Compared to ATP-competitive mTOR inhibitors, which directly bind to the mTOR catalytic site, rapamycin is an allosteric inhibitor that exclusively binds to the FKBP12/rapamycinbinding (FRB) domain of mTORC1 to restrict the access of substrates to the catalytic site. Previous phosphoproteomic studies have shown that the mTORC1 substrates, p70S6K and 4E$\mathrm{BP} 1$, are differentially sensitive to rapamycin treatment, based on the avidity with which they are phosphorylated by mTORC1. p70S6K (Thr389) and 4E-BP1 (Ser65) are weakly phosphorylated by $\mathrm{mTORC} 1$ and readily sensitive to rapamycin treatment, whereas 4E-BP1 (Thr37/46) are avidly phosphorylated and insensitive to rapamycin treatment ${ }^{41}$. In contrast, all p70S6K and 4E-BP1 sites are sensitive to ATP-competitive mTOR inhibitors $^{41}$. Several observations led us to exclude a role for mTORC1/ p70S6K in mediating the TGF- $\beta_{1}$ collagen response. First, TGF$\beta_{1}$-induced p70S6K phosphorylation was highly sensitive to rapamycin treatment, but rapamycin had no effect on either TGF- $\beta_{1}$-induced COL1A1 mRNA levels or collagen deposition. Second, the p70S6K specific inhibitor, LY2584702, did not inhibit the TGF- $\beta_{1}$ collagen response. Finally, treatment with the PDK1 inhibitor (GSK2334470) prevented TGF- $\beta_{1}$-induced p70S6K phosphorylation, but again had no effect on TGF- $\beta_{1}$-induced collagen synthesis.

Our data provides strong support that mTORC1 mediates the fibrogenic effects of TGF- $\beta_{1}$ via a $4 \mathrm{E}-\mathrm{BP} 1$-dependent mechanism. Analysis of cap-dependent translation complex formation in response to TGF- $\beta_{1}$ stimulation suggests that attenuation of $4 \mathrm{E}$ BP1 (Ser65) phosphorylation alone by rapamycin is not sufficient to block mTORC1-mediated cap-dependent translation and further that the potent effect of ATP-competitive mTOR inhibition on TGF- $\beta_{1}$-induced collagen synthesis is likely mediated via a marked inhibitory effect on the phosphorylation of multiple $4 \mathrm{E}$ $\mathrm{BP} 1$ sites. Conclusive evidence for the critical involvement of 4EBP1 was provided by data demonstrating that inducible expression of a dominant-negative $4 \mathrm{E}-\mathrm{BP} 1$ phosphomutant led to avid $4 \mathrm{E}-\mathrm{BP} 1$ binding to the $5^{\prime}$ cap and a profound inhibitory effect on the TGF- $\beta_{1}$-induced collagen response. In addition, silencing $4 \mathrm{E}$ BP1 by siRNA targeted knockdown (which effectively mimics 4EBP1 phosphorylation), rescued the inhibitory effect of the mTOR inhibitor, AZD8055, on TGF- $\beta_{1}$-induced collagen synthesis, indicating that AZD8055 exerts its anti-fibrotic effects via a 4EBP1-dependent mechanism. These findings are consistent with a recent report suggesting the involvement of mTORC1/4E-BP1 in basal collagen synthesis in the context of bronchiolitis obliterans ${ }^{40}$. However, we should caution that our findings highlighting a critical role for mTORC1/4E-BP1 conflict with a previous study reporting a role for $\mathrm{mTORC} 2$ based on Western blotting for collagen following shRNA knockdown of RICTOR ${ }^{39}$. We are currently unable to explain these contrasting findings, but it is worth commenting that we employed the most selective pharmacological tools available to probe multiple kinases along the 
PI3K/Akt/mTOR axis in combination with a highly efficient CRISPR-Cas9 gene-editing approach and used two complementary methods for assessing collagen synthesis (macromolecular crowding assays and hydroxyproline levels). Further studies using conditional tissue-specific RPTOR and RICTOR deficient mice may help resolve the issue with respect to the relative contributions of $\mathrm{mTORC} 1$ versus $\mathrm{mTORC} 2$ signaling and fibrogenesis in an in vivo setting.

In terms of identifying the mechanism by which mTORC1/4EBP1 influences the TGF- $\beta_{1}$ collagen response, several lines of investigation suggest that this occurs, at least in part, at the level of regulating COL1A1 mRNA levels. First, AZD8055 attenuated TGF- $\beta_{1}$-induced COL1A1 mRNA levels; second, the impact of AZD8055 on COL1A1 mRNA levels could be rescued by targeted $4 \mathrm{E}-\mathrm{BP} 1$ silencing; and third, inducible expression of a dominantnegative $4 \mathrm{E}-\mathrm{BP} 1$ phosphomutant blocked the increase in TGF- $\beta_{1}$ induced COL1A1 mRNA levels. Given the key role of the canonical Smad pathway in mediating downstream TGF- $\beta_{1}$ signaling, we also examined how mTORC1 signaling might intercept with the Smad pathway to regulate TGF- $\beta_{1}$-induced collagen expression. We focused our functional interrogations on Smad3, which has been strongly implicated in mediating the fibrogenic effects of TGF- $\beta_{1}$ in vitro and in vivo ${ }^{42,43}$. Our data show that Smad3 and mTORC1 signaling are both critical and cooperate to promote TGF- $\beta_{1}$-induced collagen deposition. We now propose a potential model whereby canonical Smad3 signaling influences immediateearly COL1A1 gene transcription as previously described ${ }^{42}$, but is also critical for the activation of the mTORC1/4E-BP1 axis following TGF- $\beta_{1}$ stimulation. This axis in turn is critical for TGF$\beta_{1}$-induced collagen expression, at least in part, by influencing peak COL1A1 mRNA levels, possibly by influencing the translation of an as yet unidentified protein intermediate (Fig. 6h).

Seminal ribosome profiling studies by the Sabatini laboratory have demonstrated that mRNAs with a terminal oligopyrimidine (TOP) motif in the 5'UTR (which includes ribosomal proteins and translation elongation factors) exhibit enhanced sensitivity to translational regulation by the mTORC1/4E-BP1 axis ${ }^{29}$. Sequence analysis of the 5'UTR of COL1A1 and COL1A2 mRNAs (RefSeq accession NM_000088, NM_000089, dbTSS) revealed that these transcripts do not satisfy the original criteria for TOP/TOP-like motifs. However, given the profound inhibitory effect observed by disrupting mTORC1/4E-BP1 signaling on TGF- $\beta_{1}$-induced collagen synthesis, we propose that mTORC1/4E-BP1 might act at multiple levels of the collagen biosynthetic cascade. Further extensive studies, including ribosome profiling, will be required to pinpoint the precise mechanism by which mTORC1/4E-BP1 influences TGF- $\beta_{1}$-induced collagen expression.

A number of ATP-competitive mTOR inhibitors are now reaching the clinical phase of development in other disease contexts (including equally fatal conditions such as cancer ${ }^{44}$ ), we therefore extended our analysis to determine the impact of ATPcompetitive mTOR inhibition on other TGF- $\beta_{1}$ regulated matrisome proteins in IPF-derived fibroblasts by comparing the effect of CZ415 (the most potent and selective ATP-competitive mTOR inhibitor tested, Supplementary Fig. 2) with rapamycin. Our proteomic studies revealed that there were major differences between the anti-fibrotic potential of these two compounds: in addition to inhibiting collagen I, CZ415 inhibited other major components of the fibrogenic niche including several other collagen types (III, V, VII), elastin (ELN), several matricellular proteins, and collagen processing enzymes. In contrast, the upregulation of these proteins was completely rapamycininsensitive. Of the CZ415-sensitive proteins, only ELN was classifiable as a TOP mRNA whereas COL5A1, MFAP2, and MFAP4 are classifiable as potentially TOP-like. The translation of these mRNAs may therefore be directly regulated by the mTORC1/4E-
BP1 axis, further supporting the notion that ATP-competitive mTOR inhibition acts at multiple steps to modulate the TGF- $\beta_{1}$ ECM response. We further propose that the lack of a direct antifibrogenic effect by rapamycin might, in part, explain the recent negative IPF clinical trial of the closely related rapalog, everolimus ${ }^{45}$.

Having established a basis for exploring ATP-competitive mTOR inhibition as a potential novel anti-fibrotic strategy, we focused on establishing a human-disease-based rationale for targeting this axis in the context of IPF. Support for this strategy in the most commonly used animal model of bleomycin-induced lung injury and fibrosis in mice became available during the course of this study based on the ATP-competitive mTOR inhibitor, MLN0128 39 . The bleomycin model is widely regarded to be helpful in terms of enabling mechanistic investigations relevant to fibrogenesis in an in vivo context. However, it also has wellrecognized limitations in terms of recapitulating important features of the human disease and downstream clinical predictability ${ }^{46}$. We therefore elected to further investigate the potential of ATP-competitive mTOR inhibition in IPF by investigating the impact of such an approach on collagen synthesis in live precision-cut ex-vivo lung slices derived from transplant tissue from IPF patients. These studies revealed that the potent and selective ATP-competitive mTOR inhibitor, CZ415, was highly effective at inhibiting collagen synthesis in these IPF slices. Our data further provided evidence of target pathway engagement, as the anti-fibrotic effect of CZ415 was associated with inhibition of 4E-BP1 phosphorylation at residues demonstrated throughout our study to be critical for regulation of the collagen biosynthetic cascade. Future studies with clinically developable ATPcompetitive mTOR inhibitors focused on determining their safety, efficacy, and therapeutic window in animal models of pulmonary fibrosis will be needed in order to further support the translatability of this approach for the treatment of IPF.

Excessive ECM deposition leading to architectural distortion and functional organ impairment is the hallmark of all fibrotic disease ${ }^{47}$ and is also a feature of the stromal reaction in cancer ${ }^{1}$. Most of the work presented herein focused on the collagen response in the context of IPF, the most rapidly progressive and fatal of all fibrotic diseases. However, it is recognized that although the initial insult may be distinct in terms of promoting the development of fibrosis across different organs, there are likely to be common pro-fibrotic pathways that drive ECM deposition in different disease contexts ${ }^{2}$. Data obtained using CRISPR-Cas9 gene editing of RPTOR and RICTOR in combination with data obtained by $4 \mathrm{E}-\mathrm{BP} 1$ siRNA rescue of the inhibitory effects of AZD8055 on the TGF- $\beta_{1}$ collagen response provide strong support that our key observation regarding the critical role of the mTORC1/4E-BP1 signaling during fibrogenesis is generalizable to other key effector cells of the fibrotic response in the context of the skin (pHDFs), the liver (HSCs), and the stromal reaction in lung adenocarcinoma (CAFs).

In conclusion, we report evidence based on multiple lines of investigation that the $\mathrm{mTORC} 1 / 4 \mathrm{E}-\mathrm{BP} 1$ axis plays a critical role in mediating the fibrogenic effects of TGF- $\beta_{1}$, the most potent pro-fibrotic mediator characterized to date. We propose that targeting this axis may hold broad promise as a potential antifibrotic strategy in the context of multiple fibrotic conditions and the stromal reaction in cancer.

\section{Methods}

Primary cell culture. pHLFs were grown from explant cultures of IPF or non-IPF control lung tissue. Briefly, lung parenchyma was cut into $1 \mathrm{~mm}^{3}$ fragments and placed on $10 \mathrm{~cm}$ tissue culture dishes with Dulbecco's modified Eagle's medium (DMEM) (ThermoFisher Scientific) supplemented with $10 \%(\mathrm{v} / \mathrm{v})$ fetal calf serum (FCS) (Sigma-Aldrich), penicillin $(100 \mathrm{U} / \mathrm{ml})$, streptomycin $(100 \mu \mathrm{g} / \mathrm{ml})$, 
Table 1 Guide RNAs and primer sequences used for CRISPR-Cas9 gene editing

RICTOR, exon 29

gRNA

MiSeq reverse primer
MiSeq forward primer

AATATCGGCTCATCAAATTGGGG

ACACTCTTTCCCTACACGACGctcttccgatctATGACCTACCCTCTGATGGAAAG TGACTGGAGTTCAGACGTGTGctcttccgatctTTTTTCTCTCTCAGAGATGAGGT

RAPTR, exon 26

gRNA

MiSeq forward primer

MiSeq reverse primer
CCGCGTCTACGACACAGAAGGATGG

ACACTCTTTCCCTACACGACGctcttccgatctACCCAACCAAATGGCAGTGACAC TGACTGGAGTTCAGACGTGTctcttccgatctTGCCTGTGTTTGGCTCTAGGACA and $2.5 \mu \mathrm{g} / \mathrm{ml}$ amphotericin B (ThermoFisher Scientific). Fibroblasts developed into a near confluent monolayer of cells after 3-4 weeks. Experiments were conducted on cells between passages 2 and 8 . CAF cell cultures were established through the Tracking Cancer Evolution through Therapy (TRACERx) clinical study (REC reference 13/LO/1546). CAFs were grown from explant culture of tumor tissue from patients with lung adenocarcinoma. Primary normal human adult dermal fibroblasts and human HSCs were purchased from Lonza (\#CC-2511) and Zen-Bio (\#HP-F-S), respectively.

To generate a HLF cell line expressing a dominant-negative mutant of 4E-BP1, fibroblasts were transduced with lentiviral tet-on plasmid. pCW57.1-4EBP1_4xAla was a gift from David Sabatini (Addgene plasmid \#38240)29. Cells were selected with $2 \mu \mathrm{g} / \mathrm{ml}$ puromycin (Sigma-Aldrich) for 5 days. Expression of the 4E-BP1 mutant was induced with $1 \mu \mathrm{g} / \mathrm{ml}$ doxycycline treatment (Sigma-Aldrich) for $24 \mathrm{~h}$.

For experiments analyzed by Western blot or real-time RT-PCR, cells were seeded into 6-well plates (Nunc ${ }^{\text {twm }}$, ThermoFisher Scientific) and cultured for $48 \mathrm{~h}$ prior to serum starvation. Fibroblast cultures assessed for collagen I deposition were seeded into black-walled 96-well plates (Corning) for $24 \mathrm{~h}$ prior to low-serum $(0.4 \%)$ starvation. All cell lines tested negative for mycoplasma.

Immunoblotting. Fibroblasts were lysed in ice-cold PhosphoSafe ${ }^{\circledR}$ buffer (Merck Millipore) supplemented with protease inhibitors (cOmpleteMini ${ }^{\mathrm{mt}}$, Roche). Following SDS PAGE, gel to membrane transfer and blocking, protein phosphorylation was assessed by Western blot using commercially available antibodies (catalogue numbers and dilutions supplied in Supplementary Table 1), and visualization of membranes was performed using ImageQuant TL v8.1 software (GE Healthcare). Following stripping, membranes were blocked and re-probed for total levels of specified proteins. Uncropped Western blots for all figures are shown in Supplementary Figs. 6-15.

Determination of type I collagen deposition. Type I collagen biosynthesis and deposition was determined in a 96-well format by a high-content immunofluorescence-based macromolecular crowding assay modified from a previously described method $^{27}$. Briefly, fibroblasts were cultured in DMEM $(0.4 \%$ FCS) in the presence of ascorbic acid and a dissolved mix of Ficoll 70 and Ficoll 400 as macromolecular crowding agents. For compound studies, fibroblasts were incubated either with vehicle (DMSO) or a specified inhibitor (Supplementary Table 2, Supplementary Fig. 1$)$ for $1 \mathrm{~h}$ prior to stimulation with TGF- $\beta_{1}(1 \mathrm{ng} / \mathrm{ml})$ and further incubation for $72 \mathrm{~h}$ at which point cell monolayers were fixed for immunofluorescence. Fibroblasts were stained with a collagen I antibody (SigmaAldrich) and AlexaFluor488 secondary antibody (ThermoFisher Scientific), nuclei were counterstained with DAPI. Images of fibroblasts and collagen deposition were captured and the fluorescent signal quantified on the ImageXpress Micro XLS high-content imaging system at $20 \times$ magnification (Molecular Devices).

COL1A1 real-time quantitative PCR. Total RNA was extracted from adherent cells using RNeasy Mini kit (Qiagen) according to the manufacturer's instructions. Realtime PCR was performed using a Mastercycler Realplex ep gradient S (Eppendorf). Primer sequences: COL1A1 forward 5' ATGTAGGCCACGCTGTTCTT $3^{\prime}$ and COL1A1 reverse 5' GAGAGCATGACCGATGGATT 3'. PCR amplification was carried out for 40 cycles at a melting temperature of $95^{\circ} \mathrm{C}$ for $15 \mathrm{~s}$ and an annealing temperature of $60^{\circ} \mathrm{C}$ for $1 \mathrm{~min}$. A dissociation curve was analyzed for each PCR experiment to assess primer-dimer formation or contamination. Relative mRNA level quantifications of target genes were determined using the cycle threshold method with $A T P 5 B$ and $\beta 2$ microglobulin $(\beta 2 M)$ as housekeeping genes (PrimerDesign Ltd. Primer sequences for housekeeping genes are proprietary, accession numbers supplied by manufacturer: NM_001686 (ATP5B), NM_004048 $(\beta 2 M))$, and the data were expressed as the expression relative to the housekeeping genes.

CRISPR-Cas9 gene editing. Guide RNA (gRNA) sequences were designed using the Deskgen design platform (https://www.deskgen.com/guidebook/advanced. $\mathrm{html}$ ). Primary cells were electroporated with a CRISPR ribonucleoprotein (RNP) complex targeting either RICTOR exon 29 or RPTOR exon 26 using the Lonza $4 \mathrm{D}$
Nucleofector $^{\mathrm{m}}$ system (Basel, Switzerland). MiSeq analysis was performed to genotype the loci that were targeted by the CRISPR RNP complex and showed $94 \%$ allele mutation frequency. Loss of Rictor and Raptor protein expression was confirmed using Western blotting. The gRNA sequences and primers used are given in Table 1 .

siRNA transfection. Confluent cells were serum starved and transfected with 10-50 nM ON-TARGETplus siRNA pools targeting EIF4EBP1 or SMAD3 (Dharmacon) or Silencer Select negative control siRNA (ThermoFisher Scientific) using RNAiMax lipofectamine (Invitrogen) according to the manufacturer's instructions.

$\mathbf{m}^{7}$ GTP immunoprecipitation. Fibroblasts were lysed in ice-cold Pierce immunoprecipitation lysis buffer (ThermoFisher Scientific) supplemented with EDTAfree protease and phosphatase inhibitors (ThermoFisher Scientific). Association of proteins with the $5^{\prime}$ mRNA cap was determined by first incubating lysates with $\mathrm{m}^{7}$ GTP-bound sepharose beads (Jena Bioscience), followed by Western blot to assess the association of relevant proteins.

Matrisome proteomics. IPF-derived lung fibroblasts were grown in macromolecular crowding conditions and treated with either vehicle (DMSO), rapamycin $(100 \mathrm{nM})$, or the mTOR inhibitor CZ415 $(5 \mu \mathrm{M})$. Following compound treatment for $3 \mathrm{~h}$, TGF- $\beta_{1}(1 \mathrm{ng} / \mathrm{ml})$ was added for $24 \mathrm{~h}$, and cells were harvested by scraping of the culture surface. PBS-washed cell pellets were then processed for LC-MS/MS analysis (please see Supplementary materials and methods for a detailed protocol). The mass spectrometry proteomics data have been deposited to the ProteomeXchange consortium via the PRIDE partner repository ${ }^{48}$ with dataset identifier PXD010164.

Collagen synthesis in precision-cut lung slices. To generate precision-cut lung slices, IPF tissue was first inflated with $3 \%$ agarose dissolved in RPMI and cooled. $8 \mathrm{~mm}$ diameter cores were generated and $250 \mu \mathrm{m}$ slices were produced using the Krumdieck tissue slicer (Alabama Research and Development). Slices were cultured for $24 \mathrm{~h}$ in DMEM ( $10 \% \mathrm{CO}_{2} / 100 \%$ humidity) prior to incubation with the mTOR inhibitor, CZ415, for $120 \mathrm{~h}$ (media changed and CZ415 re-added at $72 \mathrm{~h}$ ). The effect of CZ415 on levels of the collagen formation marker, P1NP, in tissue supernatant was assessed by proprietary competitive ELISA (Nordic Bioscience) ${ }^{49}$ Tissue slices were homogenized and analyzed for the phosphorylation of 4E-BP1 by MSD immunoassay (Meso Scale Discovery, USA)

Quantification of hydroxyproline from cellular supernatants. Fibroblast procollagen production was assessed by high-performance liquid chromatography (HPLC) quantitation of hydroxyproline in supernatants from confluent fibroblast monolayers. Briefly, proteins were precipitated by the addition of ethanol to a final concentration of $67 \%(\mathrm{vol} / \mathrm{vol})$ at $4{ }^{\circ} \mathrm{C}$ overnight and separated from free amino acids with $0.45 \mu \mathrm{m}$ filters (Millipore). Filters with adherent proteins were hydrolyzed in hydrochloric acid $(6 \mathrm{M})$ at $110^{\circ} \mathrm{C}$ overnight. Hydrolysates were decolorized with charcoal, filtered through a $0.65 \mu \mathrm{m}$ filter (Millipore) (Sigma Aldrich) and then derivatized with 7-chloro-4-nitrobenzo-2-oxa-1,3-diazole (Acros Organics, ThermoFisher Scientific) prior to reverse-phase HPLC (Agilent 1100 series, Agilent Technologies) for the isolation of hydroxyproline with an acetonitrile gradient using a LiChrosopher, 100 RP-18 column.

Statistical analysis. Concentration-response curves, mRNA and tissue slice data figures were constructed using GraphPad Prism version 7.00. Four-parameter nonlinear regression analyses were used to generate $\mathrm{IC}_{50}$ values from concentration-response curves. Additionally, where specified in relevant figure legends, data were analyzed by Student's $t$-test, one-way ANOVA with Dunnett's multiple-comparisons testing or two-way ANOVA with Tukey multiplecomparisons testing. Data were considered statistically significant at $p<0.05$.

Study approval. Samples of IPF lung tissue were obtained from patients undergoing lung transplant or surgical lung biopsy following informed signed consent 
and with research ethics committee approval (11/NE/0291-NRES Committee North East-Newcastle and North Tyneside 1, 10/H0504/9-National Institute of Health Biomedical Research Unit Advanced Disease Biobank, Royal Brompton Hospital, 10/H0720/12-London-Hampstead Research Ethics Committee and 12/ EM/0058-NRES Committee East Midlands-Nottingham 2). Cancer-associated fibroblast (CAF) cell cultures were established within the Tracking Cancer Evolution through Therapy (TRACERx) clinical study (13/LO/1546-NRES LondonCamden and Islington). Tissue for lung slice experiments was obtained from Asterand Europe (Royston, UK) in compliance with the UK Human Tissue Act 2004. The human biological samples were sourced ethically and their research use was in accord with the terms of the informed consents.

\section{Data availability}

The mass spectrometry proteomics data have been deposited to the ProteomeXchange consortium via the PRIDE repository, and are publicly available with the dataset identifier PXD010164. Complete selectivity profiles of CZ415 and AZD8055 are available in Supplementary Data 1. Complete matrisome and collagen-modifying protein data is available in Supplementary Data 2. All remaining data will be available from the corresponding author upon reasonable request.

Received: 20 December 2017 Accepted: 29 November 2018 Published online: 02 January 2019

\section{References}

1. Kalluri, R. The biology and function of fibroblasts in cancer. Nat. Rev. Cancer 16, 582-598 (2016)

2. Rockey, D. C., Bell, P. D. \& Hill, J. A. Fibrosis-a common pathway to organ injury and failure. N. Engl. J. Med. 372, 1138-1149 (2015).

3. Bremnes, R. M. et al. The role of tumor stroma in cancer progression and prognosis: emphasis on carcinoma-associated fibroblasts and non-small cell lung cancer. J. Thorac. Oncol. 6, 209-217 (2011).

4. King, T. E. et al. A phase 3 trial of pirfenidone in patients with idiopathic pulmonary fibrosis. N. Engl. J. Med. 370, 2083-2092 (2014).

5. Richeldi, L. et al. Efficacy and safety of nintedanib in idiopathic pulmonary fibrosis. N. Engl. J. Med. 370, 2071-2082 (2014).

6. Mora, A. L., Rojas, M., Pardo, A. \& Selman, M. Emerging therapies for idiopathic pulmonary fibrosis, a progressive age-related disease. Nat. Rev. Drug Discov. 16, 755-772 (2017).

7. Álvarez, D. et al. IPF lung fibroblasts have a senescent phenotype. Am. J. Physiol. Cell Mol. Physiol. 313, L1164-L1173 (2017).

8. Yang, I. V. \& Schwartz, D. A. Epigenetics of idiopathic pulmonary fibrosis. Transl. Res. https://doi.org/10.1016/j.trsl.2014.03.011 (2014).

9. Hinz, B. The role of myofibroblasts in wound healing. Curr. Res. Transl. Med. 64, 171-177 (2016)

10. Scotton, C. J. \& Chambers, R. C. Molecular targets in pulmonary fibrosis. Chest 132, 1311-1321 (2007)

11. Kuhn, C. \& McDonald, J. A. The roles of the myofibroblast in idiopathic pulmonary fibrosis. Ultrastructural and immunohistochemical features of sites of active extracellular matrix synthesis. Am. J. Pathol. 138, 1257-1265 (1991).

12. Novo, E. et al. Overexpression of Bcl-2 by activated human hepatic stellate cells: resistance to apoptosis as a mechanism of progressive hepatic fibrogenesis in humans. Gut 55, 1174-1182 (2006).

13. Santiago, B., Galindo, M., Rivero, M. \& Pablos, J. L. Decreased susceptibility to Fas-induced apoptosis of systemic sclerosis dermal fibroblasts. Arthritis Rheum. 44, 1667-1676 (2001).

14. Phan, S. H. Genesis of the myofibroblast in lung injury and fibrosis. Proc. Am. Thorac. Soc. 9, 148-152 (2012).

15. Kim, K. K., Sheppard, D. \& Chapman, H. A. TGF- $\beta 1$ signaling and tissue fibrosis. Cold Spring Harb. Perspect. Biol. 10, a022293 (2018).

16. Akhurst, R. J. Targeting TGF- $\beta$ signaling for therapeutic gain. Cold Spring Harb. Perspect. Biol. 9, a022301 (2017).

17. Engelman, J. A., Luo, J. \& Cantley, L. C. The evolution of phosphatidylinositol 3-kinases as regulators of growth and metabolism. Nat. Rev. Genet. 7, 606-619 (2006).

18. Laplante, M. \& Sabatini, D. mTOR signaling in growth control and disease. Cell 149, 274-293 (2012).

19. Huang, J. \& Manning, B. D. The TSC1-TSC2 complex: a molecular switchboard controlling cell growth. Biochem. J. 412, 179-190 (2008).

20. White, E. S. et al. Negative regulation of myofibroblast differentiation by PTEN (phosphatase and tensin homolog deleted on chromosome 10). Am. J. Respir. Crit. Care Med. 173, 112-121 (2006).
21. Xia, H. et al. Pathological integrin signaling enhances proliferation of primary lung fibroblasts from patients with idiopathic pulmonary fibrosis. J. Exp. Med. 205, 1659-1672 (2008).

22. Mercer, P. F. et al. Exploration of a potent PI3 kinase/mTOR inhibitor as a novel anti-fibrotic agent in IPF. Thorax 71, 701-711 (2016).

23. Bendell, J. C. et al. Phase I, dose-escalation study of BKM120, an oral panClass I PI3K inhibitor, in patients with advanced solid tumors. J. Clin. Oncol. 30, 282-290 (2012).

24. Fruman, D. A. \& Rommel, C. PI3K and cancer: lessons, challenges and opportunities. Nat. Rev. Drug Discov. 13, 140-156 (2014).

25. Heudel, P.-E. et al. Phase II study of the PI3K inhibitor BKM120 in patients with advanced or recurrent endometrial carcinoma: a stratified type I-type II study from the GINECO group. Br. J. Cancer 116, 303-309 (2017).

26. García-Martínez, J. M. \& Alessi, D. R. mTOR complex 2 (mTORC2) controls hydrophobic motif phosphorylation and activation of serum- and glucocorticoid-induced protein kinase 1 (SGK1). Biochem. J. 416, 375-385 (2008).

27. Chen, C. Z. C. et al. The Scar-in-a-Jar: studying potential antifibrotic compounds from the epigenetic to extracellular level in a single well. Br.J. Pharmacol. 158, 1196-1209 (2009).

28. Gingras, A. C. et al. Hierarchical phosphorylation of the translation inhibitor 4E-BP1. Genes Dev. 15, 2852-2864 (2001).

29. Thoreen, C. C. et al. A unifying model for mTORC1-mediated regulation of mRNA translation. Nature 485, 109-113 (2012).

30. Ishii, G., Ochiai, A. \& Neri, S. Phenotypic and functional heterogeneity of cancer-associated fibroblast within the tumor microenvironment. Adv. Drug Deliv. Rev. 99, 186-196 (2016).

31. Horowitz, J. C. et al. Combinatorial activation of FAK and AKT by transforming growth factor-betal confers an anoikis-resistant phenotype to myofibroblasts. Cell Signal. 19, 761-771 (2007).

32. Horowitz, J. C. et al. Activation of the pro-survival phosphatidylinositol 3kinase/AKT pathway by transforming growth factor-betal in mesenchymal cells is mediated by p38 MAPK-dependent induction of an autocrine growth factor. J. Biol. Chem. 279, 1359-1367 (2004)

33. Conte, E. et al. Inhibition of PI $3 \mathrm{~K}$ prevents the proliferation and differentiation of human lung fibroblasts into myofibroblasts: the role of class I P110 isoforms. PLoS ONE 6, e24663 (2011)

34. Ricupero, D. A. et al. Phosphatidylinositol 3-kinase-dependent stabilization of a1(I) collagen mRNA in human lung fibroblasts. Am. J. Physiol. Cell Physiol. 281, C99-C105 (2001).

35. Runyan, C. E., Schnaper, H. W. \& Poncelet, A.-C. The phosphatidylinositol $3-$ kinase/Akt pathway enhances Smad3-stimulated mesangial cell collagen I expression in response to transforming growth factor- $\beta 1$. J. Biol. Chem. 279, 2632-2639 (2004).

36. Cleary, J. M. \& Shapiro, G. I. Development of phosphoinositide-3 kinase pathway inhibitors for advanced cancer. Curr. Oncol. Rep. 12, https://doi.org/ 10.1007/s11912-010-0091-6 (2010).

37. Saxton, R. A. \& Sabatini, D. M. mTOR signaling in growth, metabolism, and disease. Cell 168, 960-976 (2017).

38. Mendoza, M. C., Er, E. E. \& Blenis, J. The Ras-ERK and PI3K-mTOR pathways: cross-talk and compensation. Trends Biochem. Sci. 36, 320-328 (2011)

39. Chang, W. et al. A critical role for the mTORC2 pathway in lung fibrosis. PLoS ONE 9, e106155 (2014)

40. Walker, N. M. et al. Mechanistic target of rapamycin complex 1 (mTORC1) and $\mathrm{mTORC} 2$ as key signaling intermediates in mesenchymal cell activation J. Biol. Chem. 291, 6262-6271 (2016).

41. Kang, S. A. et al. mTORC1 phosphorylation sites encode their sensitivity to starvation and rapamycin. Science 341, 1236566 (2013).

42. Verrecchia, F., Chu, M. L. \& Mauviel, A. Identification of novel TGF-beta/ Smad gene targets in dermal fibroblasts using a combined cDNA microarray/ promoter transactivation approach. J. Biol. Chem. 276, 17058-17062 (2001).

43. Bonniaud, P. et al. Smad3 null mice develop airspace enlargement and are resistant to TGF-beta-mediated pulmonary fibrosis. J. Immunol. 173, 2099-2108 (2004).

44. Xie, J., Wang, X. \& Proud, C. G. mTOR inhibitors in cancer therapy. F1000Res. 5, 2078 (2016).

45. Malouf, M. A., Hopkins, P., Snell, G. \& Glanville, A. R. An investigator-driven study of everolimus in surgical lung biopsy confirmed idiopathic pulmonary fibrosis. Respirology 16, 776-783 (2011).

46. Jenkins, R. G. et al. An Official American Thoracic Society Workshop Report: use of animal models for the preclinical assessment of potential therapies for pulmonary fibrosis. Am. J. Respir. Cell Mol. Biol. 56, 667-679 (2017).

47. Henderson, N. C. et al. Targeting of av integrin identifies a core molecular pathway that regulates fibrosis in several organs. Nat. Med. 19, 1617-1624 (2013). 
48. Vizcaíno, J. A. et al. 2016 update of the PRIDE database and its related tools. Nucleic Acids Res. 44, D447-D456 (2016).

49. Leeming, D. J. et al. Enzyme-linked immunosorbent serum assays (ELISAs) for rat and human N-terminal pro-peptide of collagen type I (PINP) assessment of corresponding epitopes. Clin. Biochem. 43, 1249-1256 (2010).

\section{Acknowledgements}

The authors thank members of the lung TRACERx consortium whose study enabled the derivation of the cancer-associated fibroblasts (CAFs) used in this study. The authors acknowledge support from the Medical Research Council UK (MR/K024078/1 to H.V. W.), the Biotechnology and Biological Research Council (BB/L502133/1 to R.C.C.), the National Institute for Health Research University College London Hospitals Biomedical Research Centre. Funding was also received via a collaborative framework agreement between GlaxoSmithKline (GSK) and University College London (R.C.C.). R.E.H. is funded by a Sir Henry Wellcome Postdoctoral Fellowship from the Wellcome Trust (209199/Z/17/Z). C.S. is a Royal Society Napier Research Professor. The TRACERx study is funded by Cancer Research UK (CRUK; C11496/A17786) and the derivation of TRACERx patient models was supported by the CRUK Lung Cancer Centre of Excellence and the Francis Crick Institute, which receives its core funding from CRUK (FC001169), the MRC (FC001169), and the Wellcome Trust (FC001169). A.J.F. acknowledges funding support via a collaborative framework agreement between GSK and Newcastle University and from the NIHR Newcastle Biomedical Research Centre (Newcastle BRC).

\section{Author contributions}

R.C.C. and P.F.M. contributed equally as senior authors and conceived, designed, and supervised the study, interpreted data and drafted and edited the manuscript. H.V.W. and J.D.E. performed experiments, analyzed and interpreted data, generated figures, drafted, reviewed and approved the final manuscript. D.G., M.P., M.M., R.B.G., E.J.F., and M.R. performed experiments, analyzed and interpreted data, generated figures, reviewed and approved the final manuscript. R.E.H. and C.S. derived and provided CAFs for experiments and reviewed and approved the final manuscript. A.R.T. carried out analysis of proteomics data and provided figures for the paper, reviewed and approved the final manuscript. C.B.N. supervised tissue slice and in vitro studies, interpreted data, and reviewed and approved the final manuscript. S.P. designed, acquired, and profiled key inhibitor tools and reviewed and approved the final manuscript. G.J., D.P., G.B., and N.Z. designed and analyzed the proteomic work, reviewed and approved the final manuscript. M.K. provided input into analysis of collagen neo-epitopes and reviewed the final version of the manuscript. T.M.M., R.P.M., and A.D.B. interpreted data and reviewed and approved the final manuscript. A.J.F. obtained ethical approvals, consented patients and provided patient tissue and reviewed and approved the final manuscript.

\section{Additional information}

Supplementary Information accompanies this paper at https://doi.org/10.1038/s41467018-07858-8.

Competing interests: This work was part-funded by a collaborative framework agreement awarded to R.C.C. by GlaxoSmithKline. In terms of potential conflict of interest, the following authors: C.B.N., M.M., S.P., G.J., D.P., R.B.G., A.R.T., G.B., A.D.B., N.Z., and R.P M. were employees of GlaxoSmithKline which has R\&D programs for IPF at the time of writing. M.K. is an employee of Nordic Biosciences with a commercial interest in proprietary assay development for biomarker discovery. T.M.M. has no declarations directly related to this manuscript. He has, however, received industry-academic research funding from GlaxoSmithKline R\&D, UCB, and Novartis and has received consultancy or speakers fees from Pellis, Astra Zeneca, Bayer, Biogen Idec, Boehringer Ingelheim, Cipla, Galapagos, GlaxoSmithKline R\&D, ProMetic, Roche (and previously InterMune), Sanofi-Aventis, and UCB. A.J.F. has no declarations directly related to this manuscript. His institution has received industry-academic research funding from GlaxoSmithKline R\&D, Genentech, Pfizer and Nuformix. His institution has received consultancy fees from Mallinckrodt Pharmaceuticals. All remaining authors declare no competing interests. All data presented have been recorded in full compliance with GSK data integrity.

Reprints and permission information is available online at http://npg.nature.com/ reprintsandpermissions/

Journal peer review information: Nature Communications thanks the anonymous reviewers for their contributions to the peer review of this work.

Publisher's note: Springer Nature remains neutral with regard to jurisdictional claims in published maps and institutional affiliations.

Open Access This article is licensed under a Creative Commons BY Attribution 4.0 International License, which permits use, sharing,
adaptation, distribution and reproduction in any medium or format, as long as you give appropriate credit to the original author(s) and the source, provide a link to the Creative Commons license, and indicate if changes were made. The images or other third party material in this article are included in the article's Creative Commons license, unless indicated otherwise in a credit line to the material. If material is not included in the article's Creative Commons license and your intended use is not permitted by statutory regulation or exceeds the permitted use, you will need to obtain permission directly from the copyright holder. To view a copy of this license, visit http://creativecommons.org/ licenses/by/4.0/.

(C) The Author(s) 2019 